\title{
Nonlinear Perturbations and Abstract Evolution Equations in General Banach Spaces (*).
}

\author{
Gabriella Dr Blasio (Roma)
}

\begin{abstract}
Summary. - Part I deals with the problem of determining sufficient conditions under which the sum of two m-accretive operators on a closed convex set $Q_{1}$ is m-accretive on $Q_{1}$. Part II is concerned with the initial value problem: $u^{\prime}+A u+g(u)=v, u(0)=u_{0}$. Applications are given to the Boltzmann equation.
\end{abstract}

\section{Introduction.}

Let $X_{1}$ be a real (possibly non reflexive) Banach space with the norm \|\|$_{1}$ and $Q_{1} \subset X_{1}$ a closed convex set. Part $I$ of this paper is primarily intended to determine sufficient conditions under which the sum of two operator which are $m$-accretive on $Q_{1}$ is $m$-accretive on $Q_{1}$. Then main result in this direction is the following: let $X_{2} \hookrightarrow X_{1}$ with the norm \|\|$_{2}$ and $Q_{2}=Q_{1} \cap X_{2}$. Let $f$ be $m$-accretive on $Q_{1}, g$ : $D_{g} \supset Q_{2} \rightarrow X_{1}$ be accretive on $Q_{1}$ and assume that there exists a family of approximating functions $\left\{g_{\beta}\right\}$ such that

(i) $g_{\beta}$ is uniformly continuous and bounded on bounded subsets of $Q_{1}$;

(ii) for each $r>0$ there exists $r^{\prime}$ independent of $\beta$ such that:

$$
\left(I+\alpha f+\alpha g_{\beta}\right)\left(Q_{2}^{r^{\prime}}\right) \supset Q_{2}^{r}
$$

where $Q_{2}^{r}=\left\{u \in Q_{2},\|u\|_{2} \leqslant r\right\}$

(iii) $\lim \left\|g_{\beta}(u)-g(u)\right\|_{1}=0$ uniformly on bounded subsets of $Q_{2}$ in $X_{2}$;

then $\overline{f+g}$ is $m$-accretive on $Q_{1}$.

We continue with some regularity results for the case in which $X_{1}$ and $X_{2}$ are supposed to be reflexive: namely we will prove that if $f$ is demiclosed then the solution of

belongs to $D_{f} \cap D_{g}$.

$$
u+\alpha(\overline{f+g})(u)=v, \quad v \in Q_{2}
$$

Finally we give an example of how the preceding theory may be used in the study of certain elliptic partial differential equations.

(*) Entrata in Redazione il 2 luglio 1975.

21 - Annali di Matematica 
Part II is devoted to the study of the initial value problem

$$
\left\{\begin{array}{l}
\frac{d u}{d t}+A u+g(u)=v \\
u(0)=u_{0}
\end{array} \quad t \in[0, T],\right.
$$

where $A$ is a linear operator such that $-A+c I$ is the infinitesimal generator of a linear contraction semigroup on $Q_{1}$ for some $c \in \boldsymbol{R}$.

We start with an existence theorem providing local strong solutions of (*) for the case in which $g$ is uniformly continuous and bounded on bounded subsets of $Q_{1}$ and the condition that $g$ is $m$-accretive on $Q_{1}$ is weakened to the following:

(i) for each $r>0$ there exists $\omega(r)$ such that $\omega(r) I+g$ is accretive on $Q_{1}^{r}$ and we have

$$
(I+\omega(r) I+\alpha g))\left(a Q_{1}^{r}\right) \supset a Q_{1}^{r}
$$

for each $a<1$.

(If $A$ is continuous then (*) has a unique strict solution.)

Furthermore we prove that if there exists $\sigma$ such that

$$
\|u\|_{1} \leqslant\|u+\alpha \sigma u+\alpha g(u)\|_{1}
$$

for each $u \in Q_{1}$ then the solution of $(*)$ exists for all $T>0$.

If $g$ is not supposed to be continuous we shall prove the following result: let $D_{g} \supset Q_{2}$ and assume that there exists a family of approximating functions $\left\{g_{\beta}\right\}$ such that

(i) $g_{\beta}$ is uniformly continuous and bounded on bounded subsets of $Q_{1}$;

(ii) for each $r>0$ there exists $\omega(r)$ such that $\omega(r) I+g_{\beta}$ is accretive on $Q_{2}^{r}$ and we have

$$
\left(I+\alpha \omega(r) I+\alpha g_{\beta}\right)\left(a Q_{2}^{r}\right) \supset a Q_{2}^{r}, \quad \alpha>0
$$

for each $a \leqslant 1$;

(iii) we have $\lim \left\|g_{\beta}(u)-g(u)\right\|_{1}=0$ uniformly on bounded subsets of $Q_{2}$ in $X_{2}$;

(iv) $-A+c I$ is the infinitesimal generator of a linear contraction semi-group on $Q_{1}$ for some $c \in \boldsymbol{R}$ and we have

$$
\|u\|_{2} \leqslant\|u+\alpha c u+\alpha A u\|_{2}, \quad \alpha>0
$$

for each $u \in D_{A}$;

then there exists a unique local solution in some generalized sense of problem (*) (if $A$ is continuous then (*) has a strong solution). 
In addition, if there exists $\sigma$ such that

$$
\|u\|_{2} \leqslant\left\|u+\alpha \sigma u+\alpha g_{\beta}(u)\right\|_{2}
$$

for each $u \in Q_{2}$, then the solution exists for all $T>0$.

We conclude with some sufficient conditions concerning regularity and give a simple illustrative example.

Finally as an application of the preceding theory we study the spatially homogeneous Boltzmann equation for the ease in which the extrinsic force is not supposed to be zero and obtain results which extend those of [2], [10] and [20].

Some of the results given in this paper were presented in [11].

\section{PART I}

\section{ABSTRACT RESULTS}

\subsection{Preliminaries.}

Let $X_{1}$ be a real Banach space with the norm \|\|$_{1}$ and let $Q_{1} \subset X_{1}$ be a closed convex set. By $G\left(X_{1}\right)$ we denote the class of multivalued functions which we view as subsets of $X_{1} \times X_{1}$. If $f \in G\left(X_{1}\right)$ we define:

$$
\begin{aligned}
& f(x)=\left\{y \in X_{1}:(x, y) \in f\right\}, \\
& D_{f}=\left\{x \in X_{x}: f(x) \neq 0\right\}, \\
& f\left(D_{f}\right)=\bigcup\left\{f(x): x \in D_{f}\right\}, \\
& D_{f}-1=f\left(D_{f}\right), \\
& f^{-1}(y)=\left\{x \in D_{f}: y \in f(x)\right\} .
\end{aligned}
$$

If $f, g \in G\left(X_{1}\right)$ and $\alpha$ is real one sets

$$
\begin{aligned}
& D_{\alpha f}=D_{f}, \\
& \alpha f(x)=\{\alpha y: y \in f(x)\}, \\
& D_{f+g}=D_{f} \cap D_{g}, \\
& (f+g)(x)=\left\{y=y_{1}+y_{2}: y_{1} \in f(x), y_{2} \in g(x)\right\} .
\end{aligned}
$$

Finally we denote by $G_{0}\left(X_{1}\right) \subset G\left(X_{1}\right)$ the class of singlevalued functions and by 
$\operatorname{Lip}\left(Q_{1}\right)$ the class of all $f \in G_{0}\left(X_{1}\right)$ such that $D_{f}=Q_{1}$ and

$$
\|f\|_{1, L}=\sup _{x \neq y}\left\|\frac{f(x)-f(y)}{x-y}\right\|_{1}<+\infty .
$$

If $f \in G\left(X_{1}\right)$ one sets

$\varrho_{Q_{1}}(f)=\left\{\alpha \in \boldsymbol{R}\right.$ and $\alpha>0:(I+\alpha f)^{-1} \in Q_{0}\left(X_{1}\right),(I+\alpha f)\left(D_{f} \cap Q_{1}\right) \supset Q_{1}$,

$$
\left.(I+\alpha f)^{-1} / Q_{1} \in \operatorname{Lip}\left(Q_{1}\right)\right\}\left(^{(1)},\right.
$$

$$
J_{Q_{1}}(\alpha, f)=(I+\alpha f)^{-1} / Q_{1}, \quad \alpha \in \varrho_{Q_{1}}(f)
$$

$\varrho_{Q_{1}}(f)$ and $J_{Q_{1}}(\alpha, f)$ are called the resolvent set and the resolvent of $f$ on $Q_{1}$.

LFma 1.1.1. - If $f \in G\left(X_{1}\right), \alpha, \beta \in \varrho_{Q_{1}}(f)$ and $\alpha \geqslant \beta$ then

$$
J_{Q_{1}}(\alpha, f)=J_{Q_{1}}(\beta, f)\left(\frac{\beta}{\alpha}+\frac{\alpha-\beta}{\alpha} J_{Q_{1}}(\alpha, f)\right) .
$$

Proof. - Let $x \in Q_{1}, y \in J_{Q_{1}}(\alpha, f)(x)$ and $u \in f(y)$ be such that $y+\alpha u=x$; we have:

$$
y+\beta u=y-\frac{\beta}{\alpha} J_{Q_{1}}(\alpha, f)(x)+\frac{\beta}{\alpha} J_{Q_{1}}(\alpha, f)(x)+\beta u=\frac{\alpha-\beta}{\alpha} y+\frac{\beta}{\alpha} x \in Q_{1}
$$

from which it follows that

$$
J_{Q_{1}}(\alpha, f)(x)=y=J_{Q_{1}}(\beta, f)(y+\beta u)=J_{Q_{1}}(\beta, f)\left(\frac{\beta}{\alpha}+\frac{\alpha-\beta}{\alpha} J_{Q_{1}}(\alpha, f)\right)(x) .
$$

\subsection{Accretive functions.}

We begin this section with some basic definitions and elementary facts. Many of the results are standard and appear in the existing literature. See, e.g. [14] and [7].

Definition 1.2.1. $-f \in G\left(X_{1}\right)$ is called accretive on $Q_{1}$ if for each $\alpha>0$ and $(x, u),(y, v) \in f$ such that $x, y \in Q_{1}$ we have

$$
\|x-y\|_{1} \leqslant\|x-y+\alpha(u-v)\|_{1} .
$$

The following lemmas hold:

LEMma 1.2.1. - Let $f \in G\left(X_{1}\right)$; if (1) is verified for $\alpha=\alpha_{0}>0$ then (1) is verified for all $\alpha \in\left[\alpha_{0},+\infty[\right.$.

(1) If $h \in G\left(X_{1}\right)$ by $h / Q_{1}$ we denote the restriction of $h$ to $Q_{1}$. 
Proof. - Let $\alpha \in\left[\alpha_{0},+\infty[\right.$ and $(x, u),(y, v) \in f$; we have

$$
\|x-y\|_{1} \leqslant\left\|x-y+\alpha_{0}(u-v)\right\|_{1} \leqslant\left\|\left(1-\frac{\alpha_{0}}{\alpha}\right)(x-y)+\alpha_{0}(u-v)\right\|_{1}+\frac{\alpha_{0}}{\alpha}\|x-y\|_{1},
$$

and this implies

$$
\|x-y\|_{1} \leqslant\left\|x-y+\frac{\alpha_{0} \alpha}{\alpha-\alpha_{0}}(u-v)\right\|_{1}
$$

Setting $\varphi(\alpha)=\left(\alpha_{0} \alpha\right) /\left(\alpha-\alpha_{0}\right)\left(\alpha>\alpha_{0}\right)$ we have $\left.\varphi(] \alpha_{0},+\infty[)=\right] \alpha_{0},+\infty[$ and the result follows.

CoRollary. - If $f$ satisfies (1) for $\left.\alpha \in] 0, \alpha_{0}\right]$ then $f$ is accretive on $Q_{1}$.

LEMMA 1.2.2. - Let $f$ satisfy (1) for $\alpha=\alpha_{0}$ and $\alpha_{0} \in \varrho_{Q_{1}}(f)$. Then $\left[\alpha_{0},+\infty\left[c Q_{Q_{1}}(f)\right.\right.$ and we have

$$
J_{Q_{1}}(\alpha, f)=J_{Q_{1}}\left(\alpha_{0}, f\right)\left(\frac{\alpha}{\alpha_{0}}-\frac{\alpha-\alpha_{0}}{\alpha_{0}} J_{Q_{1}}\left(\alpha_{0}, f\right)\right)^{-1}
$$

for all $\alpha \in] \alpha_{0},+\infty[$.

Proof. - Let $y \in Q_{1}$ and $\alpha>\alpha_{0} ;$ setting

$$
g(x)=\frac{\alpha-\alpha_{0}}{\alpha} J_{Q_{1}}\left(\alpha_{0}, f\right)(x)+\frac{\alpha_{0}}{\alpha} y, \quad x \in Q_{1}
$$

we have $g\left(Q_{1}\right) \subset Q_{1}$ and $\|g\|_{1, L} \leqslant\left(\alpha-\alpha_{0}\right) / \alpha<1$. Since $g$ is a contraction there exists a unique $x \in Q_{\text {i }}$ such that

$$
\frac{\alpha}{\alpha_{0}} x-\frac{\alpha-\alpha_{0}}{\alpha_{0}} J_{Q_{1}}\left(\alpha_{0}, f\right)(x)=y
$$

and this implies

$$
\left(\frac{\alpha}{\alpha_{0}}-\frac{\alpha-\alpha_{0}}{\alpha_{0}} J_{Q_{1}}\left(\alpha_{0}, f\right)\right)^{-1} / Q_{1} \in G_{0}\left(X_{1}\right)
$$

and

$$
\left(\frac{\alpha}{\alpha_{0}}-\frac{\alpha-\alpha_{0}}{\alpha_{0}} J_{Q_{1}}\left(\alpha_{0}, f\right)\right)^{-1}\left(Q_{1}\right) \subset Q_{1}
$$

Let $y \in Q_{1}$; by Lemma 1.2.1 there exists at last one $z \in D_{f}$ such that

$$
z+\alpha f(z) \ni y
$$


moreover it is easy to verify that $z$ satisfies (2) if and only if

$$
z+\alpha f(z) \ni \frac{\alpha}{\alpha_{0}} x-\frac{\alpha-\alpha_{0}}{\alpha_{0}} J_{Q_{2}}\left(\alpha_{0}, f\right)(x)
$$

where

$$
x=\left(\frac{\alpha}{\alpha_{0}}-\frac{\alpha-\alpha_{0}}{\alpha_{0}} J_{Q_{1}}\left(\alpha_{0}, f\right)\right)^{-1}(y)
$$

Set $\tilde{z}=J_{Q_{1}}\left(\alpha_{0}, f\right)(x) ;$ since

$$
z+\alpha f(z)=\frac{\alpha}{\alpha_{0}}\left[z+\alpha_{0} f(z)\right]-\frac{\alpha-\alpha_{0}}{\alpha_{0}} z
$$

we have that $\tilde{z}$ satisfies (3) and the result follows.

Defintmox 1.2.1. $-f \in Q\left(X_{1}\right)$ is called m-accretive on $Q_{1}$ if it is accretive on $Q_{1}$ and we have $(I+\alpha f)\left(D_{f} \cap Q_{1}\right) \supset Q_{1}$ for each $\alpha>0$.

By Lemmas 1.2.1 and 1.2.2 we get the following result:

LEMMA 1.2.3. - $f$ is $m$-accretive on $Q_{1}$ if and only if there exists $\alpha_{0}>0$ such that $\left.\left.\varrho_{Q_{1}}(f) \supset\right\rceil 0, \alpha_{0}\right]$ and we have:

$$
\begin{aligned}
& \|x-y\|_{1} \leqslant \| x-y+\alpha\left(u-v \|_{1}\right. \\
& \left.\forall \alpha \in] 0, \alpha_{0}\right] \text { and }(x, u),(y, v) \in f: x, y \in Q_{1} .
\end{aligned}
$$

REMark 1. - If $f$ is accretive on $Q_{1}$ we have $\left\|(I+\alpha f)^{-1}(x)-(I+\alpha f)^{-1}(y)\right\|_{1} \leqslant$ $\leqslant\|x-y\|_{1}$ for each $x, y \in(I+\alpha f)\left(D_{f} \cap Q_{1}\right)$ and $\alpha>0$.

THEOREM 1.2.1. - If $h$ is accretive on $Q_{1}$, then the following conditions are equivalent:

(i) there exists $\alpha_{0}>0$ such that $\overline{(I+\alpha h)\left(D_{h} \cap Q_{1}\right)} \supset Q_{1}$ for each $\left.\left.\alpha \in\right] 0, \alpha_{0}\right]$,

(ii) $\bar{h}$ is $m$-accretive on $Q_{1}$ (here $\bar{h}$ denotes the closure of $h$ in $X_{1} \times X_{1}$ ).

Assume in addition that $h \in G_{0}\left(X_{1}\right)$ and that there exists $\left\{h_{\beta}\right\}_{\beta \in[0,11} \subset G_{0}\left(X_{1}\right)$ such that $h_{\beta}$ is $m$-accretive on $Q_{1}$ and $\lim h_{\beta}(x)=h(x)$ for each $x \in D_{h}$. Then if (i) or (ii) is fulfilled we have: $\beta \rightarrow 0^{+}$

(iii) $J_{Q_{1}}(\alpha, \bar{h})(y)=\lim _{\beta \rightarrow 0^{+}} J_{Q_{1}}\left(\alpha, h_{\beta}\right)(y)$ for each $y \in Q_{1}$.

Proof. - (i) $\Rightarrow$ (ii): by the accretiveness of $h$ it follows that $\bar{h}$ is accretive thus, by Lemma 1.2.3, it suffices to show that $\left.\left.\varrho_{Q_{1}}(\bar{h}) \supset\right] 0, \alpha_{0}\right]$. Let $\left.\left.\alpha \in\right] 0, \alpha_{0}\right]$; for each $y \in Q_{1}$ there exists $\left\{\left(x_{n}, u_{n}\right)\right\} \subset h$ such that $y_{n}=x_{n}+\alpha u_{n}$ and $y_{n} \rightarrow y$. Since $\left\|x_{n}-x_{m}\right\|_{1} \leqslant$ $\leqslant\left\|y_{n}-y_{m}\right\|_{1}$ we obtain that there exists $x \in Q_{1}$ such that $x_{n} \rightarrow x$ and $x_{n}+\alpha u_{n} \rightarrow y$ so that $x \in D_{\bar{h}}$ and $x+\alpha \bar{h}(x)=y$. 
(ii) $\Rightarrow$ (i). - Let $\alpha>0$ and $y \in Q_{1}$. As we have $J_{Q_{1}}(\alpha, \bar{h})(y) \in D_{\bar{h}}$ there exists $\left\{\left(x_{n}, u_{n}\right)\right\} \subset h$ such that $x_{n} \rightarrow J_{Q_{1}}(\alpha, \bar{h})(y), x_{n}+\alpha u_{n} \rightarrow y$ and the result follows.

Finally let $h_{\beta}$ satisfy the above conditions and let (i) or (ii) be fulfilled; setting

$$
\begin{aligned}
& x=J_{Q_{1}}(\alpha, \bar{b})(y) \\
& x_{\beta}=J_{Q_{1}}\left(\alpha, h_{\beta}\right)(y)
\end{aligned} \quad \text { for } y \in Q_{1} \cap(I+\alpha h)\left(D_{h}\right)
$$

we obtain $x-x_{\beta}+\alpha h_{\beta}(x)-\alpha h_{\beta}\left(x_{\beta}\right)=\alpha h_{\beta}(x)-\alpha h(x)$, from which it follows that $\left\|x-x_{\beta}\right\|_{1} \leqslant \alpha\left\|h_{\beta}(x)-h(x)\right\|_{1} \rightarrow 0$. Since $\overline{(I+\alpha h)\left(D_{h} \cap Q_{1}\right)} \supset Q_{1}$ the conclusion follows.

Definition 1.2.2. - Let $f$ be $m$-accretive on $Q_{1}$; for each $\lambda>0$ set

$$
f_{\lambda}=\frac{1}{\lambda}\left(I / Q_{1}-J_{Q_{1}}(\lambda, f)\right)
$$

The functions $f_{2}$ are called Yosida's approximating functions on $Q_{1}$.

The following lemma collects some elementary facts about the functions $f_{\lambda}$ :

Lemma 1.2.4. - If $f$ is $m$-aceretive on $Q_{1}$, then the following statements hold:

(i) if $x \in Q_{1}$ we have $f_{\lambda}(x) \in f J_{Q_{1}}(\lambda, f)(x)$;

(ii) $f_{\lambda} \in \operatorname{Lip}\left(Q_{1}\right)$ and $\left\|f_{\lambda}\right\|_{1, L} \leqslant 2 / \lambda$;

(iii) $f_{\lambda}$ is $m$-accretive on $Q_{1}$;

(iv) if $x \in D_{f}$ we have $\left\|f_{k}(x)\right\|_{1} \leqslant \inf \left\{\|y\|_{1}, y \in f(x)\right\}$.

Proof. - Assertions (i) and (ii) are elementary. In order to prove (iii) observe that

$$
\left\|J_{Q_{1}}(\lambda, f)\right\|_{1, L} \leqslant 1
$$

implies that for each $y \in Q_{1}$ there exists a unique $x \in Q_{1}$ such that

$$
x-\frac{\alpha}{\lambda+\alpha} J_{Q_{1}}(\lambda, f)(x)=\frac{\lambda}{\lambda+\alpha} y,
$$

so $\left(I+\alpha f_{\lambda}\right)\left(Q_{1}\right) \supset Q_{1}$ is proved. Now let $x_{1}, x_{2} \in Q_{1}$; setting

$$
\begin{aligned}
& x_{1}+\alpha f_{\lambda}\left(x_{1}\right)=y_{1} \\
& x_{2}+\alpha f_{\lambda}\left(x_{2}\right)=y_{2}
\end{aligned}
$$


we have

$$
\left\|x_{1}-x_{2}\right\|_{1} \leqslant \frac{\lambda}{\lambda+\alpha}\left\|y_{1}-y_{2}\right\|_{1}+\frac{\alpha}{\lambda+\alpha}\left\|y_{1}-y_{2}\right\|_{1}
$$

from which it follows that $f_{\lambda}$ is accretive. Finally we show (iv). If $x \in D_{f}$ and $u \in f(x)$ we have

$$
\left\|x-J_{Q_{1}}(\lambda, f)(x)\right\|_{1} \leqslant\left\|(I+\lambda f)^{-1}(x+\lambda u)-(I+\lambda f)^{-1}(x)\right\|_{1} \leqslant\|u\|_{1} \lambda
$$

Since $u \in f(x)$ was arbitrary, the result follows

Lemma 1.2.5. - If $f \in G_{0}\left(X_{1}\right)$ is continuous and $m$-accretive on $Q_{1}$ then

(i) $\lim _{\lambda \rightarrow 0^{+}} f_{\lambda}(x)=f(x)$ for each $x \in D_{f}$.

Moreover if $f$ is uniformly continuous and bounded on bounded subsets of $Q_{1}$ then the limit (i) is uniform on bounded subsets of $Q_{1}$.

Proof. - Using Lemma 1.2.4 (i) we have

$$
\left\|f(x)-f_{\lambda}(x)\right\|_{1}=\left\|f(x)-f J_{Q_{1}}(\lambda, f)(x)\right\|_{1}
$$

and the result follows from (4).

Theorem 1.2.2. - If $f$ and $g$ are $m$-accretive on $Q_{1}$ then $f+g$, is $m$-accretive on $Q_{1}$ and we have

$$
J_{Q_{1}}\left(\alpha, f+g_{\lambda}\right)=J_{Q_{1}}\left(\frac{\alpha \lambda}{\alpha+\lambda}, f\right)\left(\frac{\alpha+\lambda}{\lambda} I-\frac{\alpha}{\lambda} J_{Q_{1}}(\lambda, g) J_{Q_{1}}\left(\frac{\alpha \lambda}{\alpha+\lambda}, f\right)\right)^{-\lambda} .
$$

Proof. - Let $\alpha>0$; by assumption

$$
\frac{\alpha}{\alpha+\lambda} J_{Q_{1}}(\lambda, g) J_{Q_{1}}\left(\frac{\alpha \lambda}{\alpha+\lambda}, f\right)
$$

is a contraction, so there exists a unique $z \in Q_{1}$ such that

$$
z-\frac{\alpha}{\alpha+\lambda} J_{Q_{1}}(\lambda, g) J_{Q_{1}}\left(\frac{\alpha \lambda}{\alpha+\lambda}, f\right)(z)=\frac{\lambda}{\alpha+\lambda} y, \quad y \in Q_{1}
$$

Setting $x=J_{Q_{1}}(\alpha \lambda /(\alpha+\lambda), f)(z)$ we have $x \in D_{f}$ and

$$
x+\frac{\alpha \lambda}{\alpha+\lambda} f(x)-\frac{\alpha}{\alpha+\lambda} J_{Q_{1}}(\lambda, g)(x) \ni \frac{\lambda}{\alpha+\lambda} y
$$

from which it follows that $x+\alpha f(x)+\alpha g_{\lambda}(x) \ni y$ and $\left(I+\alpha f+\alpha g_{\lambda}\right)\left(D_{f}\right) \supset Q_{1}$. 
Moreover we have

$$
x=J_{Q_{1}}\left(\frac{\alpha \lambda}{\alpha+\lambda}, f\right)\left(\frac{\alpha+\lambda}{\lambda} I-\frac{\alpha}{\lambda} J_{Q_{1}}(\lambda, g) J_{Q_{1}}\left(\frac{\alpha \lambda}{\alpha+\lambda}, f\right)\right)^{-1}(y) .
$$

Now let $\left(x_{1}, u_{1}\right),\left(x_{2}, u_{2}\right) \in f ;$ setting

$$
x_{1}+\alpha u_{1}+\alpha g_{\lambda}\left(x_{1}\right)=y_{1}, \quad x_{2}+\alpha u_{2}+\alpha g_{\lambda}\left(x_{2}\right)=y_{2}
$$

and

$$
\begin{aligned}
& x_{1}+\frac{\alpha \lambda}{\alpha+\lambda} u_{1}=z_{1}, \\
& x_{2}+\frac{\alpha \lambda}{\alpha+\lambda} u_{2}=z_{2},
\end{aligned}
$$

we have

$$
\begin{aligned}
& z_{1}-\frac{\alpha}{\alpha+\lambda} J_{Q_{1}}(\lambda, g)\left(I+\frac{\alpha \lambda}{\alpha+\lambda} f\right)^{-1}\left(z_{1}\right)=\frac{\lambda}{\alpha+\lambda} y_{1}, \\
& z_{2}-\frac{\alpha}{\alpha+\lambda} J_{Q_{1}}(\lambda, g)\left(I+\frac{\alpha \lambda}{\alpha+\lambda} f\right)^{-1}\left(z_{2}\right)=\frac{\lambda}{\alpha+\lambda} y_{2}
\end{aligned}
$$

Thus $\left\|x_{1}-x_{2}\right\|_{1} \leqslant\left\|z_{1}-z_{2}\right\|_{1}$ and

$$
\left\|z_{1}-z_{2}\right\|_{1} \leqslant \frac{\lambda}{\alpha+\lambda}\left\|y_{1}-y_{2}\right\|_{1}+\frac{\alpha}{\lambda+\alpha}\left\|z_{1}-z_{2}\right\|_{1}
$$

combine to yield $\left\|x_{1}-x_{2}\right\|_{1} \leqslant\left\|y_{1}-y_{2}\right\|_{1}$ and the proof is complete.

THEOREM 1.2.3. - Let $f$ and $g$ be $m$-accretive on $Q_{1}$; if $g$ is uniformly continuous and bounded on bounded subsets of $Q_{1}$ then $\bar{f}+g$ is $m$-accretive on $Q_{1}{ }^{(2)}$.

Proof. - By Theorem 1.2.2 $f+g_{\lambda}$ is accretive, hence by Lemma 1.2.5, $f+g$ is accretive on $Q_{1}$. Now let $\alpha>0$ and $y \in Q_{1}$ and consider the set

$$
\left.\left.\left\{J_{Q_{1}}\left(\alpha, f+g_{\lambda}\right)(y), \lambda \in\right] 0,1\right]\right\}
$$

Setting $x_{1}=J_{Q_{1}}\left(\alpha, f+g_{1}\right)(y), x=J_{Q_{1}}\left(\alpha, f+g_{\lambda}\right)(y)$ we have

$$
x_{1}-x+\alpha\left[f\left(x_{1}\right)-f(x)+g_{\lambda}\left(x_{1}\right)-g_{\lambda}(x)\right] \ni \alpha\left[g_{\lambda}\left(x_{1}\right)-g_{1}\left(x_{1}\right)\right]
$$

hence by the accretiveness of $f+g_{\lambda}$ and Lemma 1.2.4 (ii)

$$
\left\|x-x_{1}\right\|_{1} \leqslant \alpha\left\|g_{\lambda}\left(x_{1}\right)-g_{1}\left(x_{1}\right)\right\|_{1} \leqslant 2 \alpha\left\|g\left(x_{1}\right)\right\|_{1}
$$

$\left.{ }^{2}\right)$ In [1] BARBU obtains a similar result in case $Q_{1}=X_{1}$. 
which implies that (5) is bounded. Using Lemma 1.2.5 and

$$
(I+\alpha f+\alpha g) J_{Q_{1}}\left(\alpha, f+g_{\lambda}\right)(y) \ni y+\alpha\left(g-g_{\lambda}\right) J_{Q_{1}}\left(\alpha, f+g_{\lambda}\right)(y)
$$

we obtain that $(I+\alpha f+\alpha g)\left(D_{f} \cap D_{g} \cap Q_{1}\right)$ is dense in $Q_{1}$. Setting $h=\bar{f}+g$ we have $\bar{h}=h$ and the result follows from Theorem 1.2.1.

\subsection{Regular functions.}

Definimion 1.3.1. $-f \in G_{0}\left(X_{1}\right)$ is called regular on $Q_{1}$ if there exists $\left\{f_{\beta}\right\} \subset G_{0}\left(X_{1}\right)$, $\beta \in] 0,1]$, such that

(i) $f_{\beta}$ is uniformly continuous and bounded on bounded subsets of $Q_{1}$;

(ii) $f_{\beta}$ is $m$-accretive on $Q_{1}$;

(iii) we have $\left\|f_{\beta}(x)\right\|_{1} \leqslant\|f(x)\|_{1}$ and $\lim f_{\beta}(x)=f(x)$ for each $x \in D_{f}$.

If $f$ is regular on $Q_{1}$ and $f_{\beta}$ satisfies (i), (ii) and (iii) the functions $f_{\beta}$ are called approximating functions of $f$ on $Q_{1}$.

REMARK 2. - It is easy to verify that if $f_{\beta} \in \operatorname{Lip}\left(Q_{1}\right), f_{\beta}$ is accretive on $Q_{1}$ and there exists $\alpha:] 0,1] \rightarrow \boldsymbol{R}^{+}, \beta \rightarrow \alpha(\beta)$ such that $\left(I-\alpha f_{\beta}\right)\left(Q_{1}\right) \subset Q_{1}$ for each $\left.\left.\alpha \in\right] 0, \alpha(\beta)\right]$ then assertions (i) and (ii) are fulfilled. In fact let $y \in Q_{1}$ and $\left.\left.\alpha \in\right] 0, \alpha(\beta)\right]$; if $\alpha\left\|f_{\beta}\right\|_{1, L}<1$ there exists a unique $x \in Q_{1}$ such that $x=\frac{1}{2} y+\frac{1}{2}\left(x-\alpha f_{\beta}(x)\right)$ and the result follows from Lemma 1.2.3.

REMARK 3. - If $f$ is $m$-accretive and continuous on $Q_{1}$ then $f$ is regular on $Q_{1}$. In fact it is easy to verify (see Remark 2 and Lemma 1.2.5) that $f_{\lambda}$ satisfy (i), (ii) and (iii) of Definition 1.3.1.

By Theorem 1.2.3 we have

THEOREM 1.3.1. - Let $f$ be $m$-accretive on $Q_{1}$ and $g$ be regular on $Q_{1}$, if $g_{\beta}$ are approximating functions of $g$ on $Q_{1}$ then $f+g_{\beta}$ is $m$-accretive on $Q_{1}$.

Theorem 1.3.2. - Let $f$ be $m$-accretive on $Q_{1}$ and $g$ be regular on $Q_{1}$. The following conditions are equivalent:

(i) there exists $\alpha_{0}>0$ such that $\overline{(I+\alpha f+\alpha g)\left(D_{f} \cap D_{g} \cap Q_{1}\right)} \supset Q_{1}$ for each $\alpha \in$ $\left.\in] 0, \alpha_{0}\right]$,

(ii) $\overline{f+g}$ is $m$-accretive on $Q_{1}$.

Moreover if (i) or (ii) is fulfilled and $g_{\beta}$ are approximating functions of $g$ we have

$$
\lim _{\beta \rightarrow 0^{+}} J_{Q}\left(\alpha, \bar{f}+g_{\beta}\right)(y)=J_{Q_{1}}(\alpha, \overline{f+g})(y) .
$$


Proof. - Theorem 1.3.1 and Definition 1.3.1 show that $f+g$ is accretive; thus, setting $h=f+g$ the assertion (i) $\Leftrightarrow$ (ii) follows from Theorem 1.2.1. The last argument in the proof of Theorem 1.2.1 can be repeated here and the proof is complete.

Let $X_{2} \subset X_{1}$ be a Banach space with the norm \|\|$_{2}$ and let $Q_{2} \subset X_{2}$ be a closed convex set dense in $Q_{1}$. The following theorem provides conditions which are sufficients to guarantee that assumption (i) of Theorem 1.3.2 holds.

Theorem 1.3.3. - Let $f \in G\left(X_{1}\right)$ and $g \in G_{0}\left(X_{1}\right)$ such that $D_{g} \supset Q_{2}$. If the following assumptions hold:

(i) there exists $\left\{g_{\beta}\right\} \subset Q_{0}\left(X_{1}\right)$ such that $D_{g_{\beta}} \supset Q_{2}$ and $\lim g_{\beta}(x)=g(x)$ uniformly on bounded subsets of $Q_{2}$ in $X_{2}$;

(ii) there exist $\alpha_{0}>0$ and a function $k:[0,+\infty[\rightarrow[0,+\infty[$ such that for each $y \in Q_{2}$ and $\left.\left.\beta \in\right] 0,1\right]$ there is $\left(x_{\beta}, u_{\beta}\right) \in f$ satisfying

$$
\left.\left.x_{\beta}+\alpha u_{\beta}+\alpha g_{\beta}\left(x_{\beta}\right)=y, \quad \alpha \in\right] 0, \alpha_{0}\right], \quad x_{\beta} \in Q_{2}
$$

and

$$
\left\|x_{\beta}\right\|_{2} \leqslant k\left(\|y\|_{2}\right)
$$

Then $\overline{(I+\alpha f+\alpha g)\left(D_{f} \cap D_{g} \cap Q_{1}\right)} \supset Q_{1}$.

Proof. - Let $\in \in Q_{2}$ and $\left(x_{\beta}, u_{\beta}\right)$ satisfying (ii); we have:

$$
x_{\beta}+\alpha u_{\beta}+\alpha g\left(x_{\beta}\right)=y+\alpha\left(g-g_{\beta}\right)\left(x_{\beta}\right)
$$

hence using (i)

$$
\lim \left(x_{\beta}+\alpha u_{\beta}+\alpha g\left(x_{\beta}\right)\right)=y .
$$

Since $X_{2}$ is dense in $X_{1}$ the result follows.

Theorem 1.3.3 extends a similar result for the case $Q_{1}=X_{1}$ and $f$ and $g$ linear. See [8].

Definttion 1.3.2. $-x \in X_{1}$ is called a strong solution of the problem

$$
x+\alpha f(x)+\alpha g(x) \ni y, \quad y \in Q_{1}
$$

if there exists $\left(x_{\beta}, u_{\beta}\right) \in f+g$ such that $x_{\beta} \rightarrow x$ and $x_{\beta}+\alpha u_{\beta} \rightarrow y$.

By Theorems 1.3.2 and 1.3.3 we get the following results:

THeorem 1.3.4. - Let $f$ be $m$-accretive and $g$ be regular on $Q_{1}$. If assumption (i) of Theorem 1.3.2 holds and $g_{\beta}$ are approximating functions of $g$ then (6) has a unique strong solution $x=\lim _{\beta \rightarrow 0^{+}} J_{Q_{1}}\left(\alpha, \bar{f}+g_{\beta}\right)(y)$. 
THeOREM 1.3.5. - Let $f$ and $g$ be accretive on $Q_{1}$ and let the assumptions of Theorem 1.3.3 hold. Then (6) has a unique strong solution $x$; moreover if $y \in Q_{2}$ we have $x=\lim _{\beta \rightarrow 0^{+}} J_{Q_{1}}\left(\alpha, f+g_{\beta}\right)(y)$.

Proof. - Let $y \in Q_{2}$ and let $\left(x_{\beta}, u_{\beta}\right)$ satisfy condition (ii) of Theorem 1.3.3; we have

$$
x_{\beta}-x_{\gamma}+\alpha\left(u_{\beta}-u_{\gamma}\right)+\alpha\left[g_{\beta}\left(x_{\beta}\right)-g_{\beta}\left(x_{\gamma}\right)\right]=\alpha\left[g_{\gamma}\left(x_{\gamma}\right)-g_{\beta}\left(x_{\gamma}\right)\right] .
$$

Hence, using Theorem 1.3 .3 (i), there exists $x=\lim _{\beta \rightarrow 0^{+}} x_{\beta}$ and the result follows.

\section{4. $M$-accretive functions in reflexive Banach spaces.}

In this section we require $X_{1}$ and $X_{2}$ to be reflexive Banach spaces.

Definition 1.4.1. $-f \in G\left(X_{1}\right)$ is called demiclosed if the following statement hold

(i) if $\left\{x_{n}\right\} \subset D_{f}, y_{n} \in f\left(x_{n}\right), x_{n} \rightarrow x$ and $y_{n} \rightarrow y$ ) $\in f$ (here $\rightarrow$ refers to convergence in the weak topology).

THEOREM 1.4.1. - Let $f$ and $g$ be accretive functions satisfying the assumptions of Theorem 1.3.3. If in addition:

(i) $f$ is demiclosed;

(ii) $\|g(x)\|_{1}$ is uniformly bounded on bounded subsets of $Q_{2}$ in $X_{2}$;

then for any $y \in Q_{2}(6)$ has a unique strong solution $x$ and we have $x=$ $\lim _{\beta \rightarrow 0^{+}} J_{Q_{1}}\left(\alpha, f+g_{\beta}\right)(y)$ and $x \in D_{f} \cap Q_{2}$.

Proof. - Let $y \in Q_{2}$ and $x_{\beta}=J_{Q_{1}}\left(\alpha, f+g_{\beta}\right)(y)$; by Theorem 1.3.5 $x=\lim _{\beta \rightarrow 0^{+}} x_{\beta}$ is the strong solution of $(6)$ and we have $\left\|x_{\beta}\right\|_{2} \leqslant k\left(\|y\|_{2}\right)$.

Since $\left\{x_{\beta}\right\}$ is bounded in $X_{2}$ there exists $\left\{x_{\beta_{k}}\right\}$ such that $x_{\beta_{k}}-\tilde{x}$ in $X_{2}$; therefore we have $\tilde{x}=x$ and $\tilde{x} \in Q_{2}$. Now let $u_{\beta} \in f\left(x_{\beta}\right)$ be such that $x_{\beta}+\alpha u_{\beta}+\alpha g_{\beta}\left(x_{\beta}\right)=y$; by assumption $\left\{g_{\beta}\left(x_{\beta}\right)\right\}$ is bounded in $X_{1}$ so that $\left\{u_{\beta}\right\}$ is bounded in $X_{1}$. It follows that there exists $\left\{u_{\beta_{h}}\right\}$ such that $u_{\beta_{k}} \rightarrow u$ hence, by the demiclosedeness of $f,(x, u) \in f$.

CoROLLARY. - In addition to the condition of Theorem 1.4.1 suppose that $g$ is demiclosed. Then for any $y \in Q_{2}(6)$ has a unique strict solution $x=\lim _{\beta \rightarrow 0^{+}} J_{Q_{1}}\left(\alpha, f+g_{\beta}\right)(y)$.

\subsection{Evolution equations.}

Let $Y_{1}$ be a real Banach space with the norm $\|_{1}$ and let $K_{1} \subset Y_{1}$ be a closed convex set. By $X_{1}^{p}$ we denote the Banach space $L^{p}\left(0, T ; Y_{1}\right)(1 \leqslant p<+\infty)$ with the usual norm \|\| and by $W^{1, p}\left(0, T ; Y_{1}\right)$ we denote the Sobolev space of all functions in $X_{1}^{p}$ all of whose distributional derivatives also belong to $X_{1}^{p}$. 
Let $g \in G\left(Y_{1}\right)$ and $w \in X_{1}^{p}$; as an application of the results obtained in Section 1.3 we shall study the initial value problem

$$
\begin{cases}\frac{d u}{d t}+g(u) \ni w & t \in[0, T], \\ u(0)=u_{0} & u_{0} \in K_{1} .\end{cases}
$$

Definition 1.5.1. - A function $u \in X_{1}^{p}$ is called a strong solution of (7) if there exists $\left\{u_{\beta}\right\}$ such that

(i) $u_{\beta} \in W^{1, p}\left(0, T ; Y_{1}\right), u_{\beta}(0)=u_{0}, u_{\beta} \rightarrow u$;

(ii) $u_{\beta}(t) \in D_{g}$ for a.e. $\left.t \in\right] 0, T[$;

(iii) there exists $\left\{v_{\beta}\right\} \subset X_{1}^{p}$ such that $v_{\beta}(t) \in g\left(u_{\beta}(t)\right)$ for a.e. $\left.t \in\right] 0, T[$ and

$$
\frac{d u_{\beta}}{d t}+v_{\beta} \rightarrow w
$$

Set $Q_{1}^{p}=\left\{u \in X_{1}^{p}, u(t) \in K_{1}\right.$ and $|u(t)|_{1} \leqslant M$ a.e. $\left.t \in\right] 0, T[\}$ where $M>\left|u_{0}\right|_{1}$, and

$$
\left\{\begin{array}{l}
D_{f^{p}}=\left\{u \in W^{1, p}\left(0, T ; Y_{1}\right), u(0)=u_{0}\right\} \\
f^{p}(u)=\frac{d u}{d t}
\end{array}\right.
$$

If $g$ is regular on $K_{1}$ with approximating functions $\left\{g_{\beta}\right\}$ we define

$$
\left\{\begin{array}{l}
D_{\tilde{g}^{p}}=\left\{u \in X_{1}^{p}, u(t) \in D_{g} \text { a.e. } t \in\right] 0, T\left[, t \rightarrow g(u(t)) \in X_{1}^{p}\right\} \\
\left(\tilde{g}^{p}(u)\right)(t)=g(u(t))
\end{array}\right.
$$

and

$$
\left\{\begin{array}{l}
D_{\tilde{g}_{\beta}^{p}}=\left\{u \in X_{1}^{p}, t \rightarrow g_{\beta}(u(t)) \in X_{1}^{p}\right\} \\
\left(\tilde{g}_{\beta}^{p}(u)\right)(t)=g_{\beta}(u(t))
\end{array}\right.
$$

The following lemma holds:

LEMMA 1.5.1. - Let $g$ be regular on $K_{1}$ with approximating functions $\left\{g_{\beta}\right\}$; if $g(0) \ni 0$ and $g_{\beta}$ is Lipschitz continuous on bounded subsets of $K_{1}$ then $\tilde{g}^{p}$ is regular on $Q_{1}^{p}$ with approximating functions $\left\{\tilde{g}_{\beta}^{p}\right\}$.

Proof. - Since $g_{\beta}$ is continuous and bounded the function $t \rightarrow g_{\beta}(u(t))$ is measurable and we have

$$
\int_{0}^{T}\left|g_{\beta}(u(t))\right|_{1}^{p} d t<+\infty, \quad u \in Q_{1}^{p}
$$


so that $D_{\tilde{g}_{\beta}^{p}} \supset Q_{1}^{p}$. Next we show that for each $\alpha>0\left(I+\alpha g_{\beta}\right)\left(Q_{1}^{p}\right) \supset Q_{1}^{p}$. Let $v \in Q_{1}^{p}$, for a.e. $t \in] 0, T\left[\right.$ there exists $u(t) \in K_{1}$ such that

$$
u(t)=\left(I+\alpha g_{\beta}\right)^{-1}(v(t))
$$

hence $u \in Q_{1}^{p}$ by the Lipschitz continuity of $\left(I+\alpha g_{\beta}\right)^{-1}$ and the assumption $g(0) \ni 0$. Since the the accretiveness of $\tilde{g}_{\beta}^{p}$ on $Q_{1}^{p}$ is evident we have that $\left\{\tilde{g}_{\beta}^{p}\right\}$ satisfies (ii) of Definition 1.3.1. It remains to verify (i) and (ii). It follows directly from the Lipschitz continuity of $g_{\beta}$ on bounded subsets of $K_{1}$ that $\tilde{g}_{\beta}^{p}$ satisfies (i). Moreover we have

$$
\lim _{\beta \rightarrow 0^{+}}\left|g_{\beta}(u(t))-g(u(t))\right|_{1}=0, \quad u \in D_{\tilde{g}^{p}}
$$

and

$$
\left|g_{\beta}(u(t))\right|_{1} \leqslant|g(u(t))|_{1}
$$

a.e. $t \in] 0, T[$. Hence using the dominated convergence theorem the conclusion follows.

REMARK 4. - It can be easily checked that the conclusion of Lemma 1.5.1 can be established without requiring the condition $g(0) \ni 0$ in the case $g_{\beta} \in \operatorname{Lip}\left(K_{1}\right)$ and replacing $Q_{1}^{p}$ by

$$
\widetilde{Q}_{1}^{p}=\left\{u \in X_{1}^{p}, u(t) \in K_{1} \text { a.e. } t \in\right] 0, T[\} .
$$

By the $m$-accretiveness of $f^{p}$ on $Q_{1}^{p}$ and by Theorem 1.3.4 we get the following results

THEOREM 1.5.1. - In addition to the conditions of Theorem 1.3.4 suppose that

$$
\left(I+\alpha_{0} f^{p}+\alpha_{0} \tilde{g}_{\beta}^{p}\right)\left(D_{f^{p}} \cap D_{\tilde{g}^{p}} \cap Q_{1}^{p}\right) \supset Q_{1}^{p}
$$

for some $\alpha_{0}>0$. Then for any $\alpha>0$ and $v \in Q_{1}^{p}$ there exists a unique strong solution of

$$
u+\alpha f^{p}(u)+\alpha \tilde{g}^{p}(u)=v
$$

and we have $u=\lim _{\beta \rightarrow 0^{+}} J_{Q_{1}}\left(\alpha, f^{p}+\tilde{g}_{\beta}^{p}\right)(v)$.

THEOREM 1.5.2. - Let the assumptions of Theorem 1.5.1 hold; if $w \in L^{p}\left(0, T ; K_{1}\right)$ then (8) has a unique strong solution.

Proof. - If $w \in L^{\infty}\left(0, T ; K_{1}\right)$ it suffices to take $M>\|w\|_{\infty}$ and the result follows from Theorem 1.5.1. Since $L^{\infty}\left(0, T ; K_{1}\right)$ is dense in $L^{p}\left(0 ; T ; K_{1}\right)$ the conclusion follows.

Now let $Y_{2} \hookrightarrow Y_{1}$ be a real Banach space with the norm $\|_{2}$ and let $K_{2} \subset Y_{2}$ be a closed convex set dense in $K_{1}$. Set $X_{2}=L^{\infty}\left(0, T ; Y_{2}\right), \widetilde{Q}_{1}^{p}=L^{p}\left(0, T ; K_{1}\right), Q_{2}=$ 
$=L^{\infty}\left(0, T ; K_{2}\right)$ and for $\left\{g_{\beta}\right\} \subset G_{0}\left(Y_{1}\right)$

$$
\left\{\begin{array}{l}
D_{\tilde{g}_{\beta}^{p}}=\left\{u \in X_{1}^{p}, u(t) \in D_{g_{\beta}} \text { a.e. } t \in\right] 0, T\left[, t \rightarrow g_{\beta}(u(t)) \in X_{1}^{p}\right\}, \\
\left(\tilde{g}_{\beta}^{p}(u)\right)(t)=g_{\beta}(u(t)) .
\end{array}\right.
$$

The following lemma holds

Lemra 1.5.2. - Let $D_{g} \supset K_{2}$ and let $|g(u)|_{1}$ be uniformly bounded on bounded subsets of $K_{2}$ in $Y_{2}$. If there exists $\left\{g_{\beta}\right\} \subset G_{0}\left(Y_{1}\right)$ such that

(i) $D_{g_{\beta}}=K_{1}, g_{\beta}$ is continuous;

(ii) we have $\lim \left|g_{\beta}(u)-g(u)\right|_{1}=0$ uniformly on bounded subsets of $K_{2}$ in $Y_{2}$;

then $D_{\tilde{g}^{p}}, D_{\tilde{g}_{\beta}^{p}} \supset Q_{2}$ and we have

(iii) $\lim \left\|\tilde{g}_{\beta}^{p}(u)-\tilde{g}^{p}(u)\right\|_{1}=0$

uniformly on bounded subsets of $Q_{2}$ in $X_{2}$.

Proof. - If $u \in Q_{2}$ we have $u(t) \in K_{2}$ and $|u(t)|_{2} \leqslant\|u\|_{\infty}$ for a.e. $\left.t \in\right] 0, T[$.

Using (i) and (ii) we then obtain that $t \rightarrow g_{\beta}(u(t))$ and $t \rightarrow g(u(t))$ are measurable and $\left|g_{\beta}(u(t))\right|_{2} \leqslant k,|g(u(t))|_{1} \leqslant k$ for a.e. $\left.t \in\right] 0, T\left[\right.$ so that $u \in D_{\tilde{g}^{p}} \cap D_{\tilde{g}_{\beta}^{p}}$. Assertion (iii) follows by noting that if $u$ belongs to a bounded subset of $Q_{2}$ then $u(t)$ belongs to a bounded subset of $K_{2}$ for a.e. $\left.t \in\right] 0, T[$.

As a consequence of Lemma 1.5.2 and Theorem 1.3.5 we get the following result:

THEOREM 1.5.3. - Let the assumptions of Lemma 1.5 .2 hold and $g$ be accretive on $K_{1}$. If in addition for each $\left.\beta \in\right] 0,1\left[\right.$ and $v \in Q_{2}$ there exists $u_{\beta} \in D_{f^{m}} \cap Q_{2}$ such that

$$
u_{\beta}+\alpha_{0} f\left(u_{\beta}\right)+\alpha_{0} \tilde{g}_{\beta}^{p}\left(u_{\beta}\right)=v
$$

and $\left\|u_{\beta}\right\|_{2} \leqslant k\left(\|v\|_{2}\right)$ for some $\alpha_{0}>0$, then there is a unique strong solution of the equation

$$
u+\alpha f^{p}(u)+\alpha \tilde{g}^{g}(u)=v, \quad v \in Q_{1} .
$$

Moreover if $v \in Q_{2}$ we have $u=\lim _{\beta \rightarrow 0^{+}} \mathcal{U}_{Q_{1}}\left(\alpha, f+\tilde{g}_{\beta}^{p}\right)(v)$.

Theorem 1.5.4. - If the assumptions of Theorem 1.5.3 hold and $Y_{1}$ and $Y_{2}$ are reflexive then for each $v \in Q_{2}$ we have $\lim _{\beta \rightarrow 0^{+}} J_{Q_{1}}\left(\alpha_{4} f+\tilde{g}_{\beta}^{p}\right)(v) \in D_{f^{p}} \cap L^{p}\left(0, T ; Y_{2}\right)(1<$ $<p<+\infty)$.

Proof. - For $v \in Q_{2}$ set

$$
u_{\beta}=J_{Q_{2}}\left(\alpha, f+\tilde{g}_{\beta}^{p}\right)(v), \quad u=\lim u_{\beta}
$$

by Theorem 1.5.3 we have $u_{\beta}(t) \in K_{2}$ and $\left|u_{\beta}(t)\right|_{2} \leqslant k(\|v\|)_{2}$ for a.e. $\left.t \in\right] 0, T[$ so that 
$\left\{u_{\beta}\right\}$ is bounded in $L^{p}\left(0, T ; Y_{2}\right)$. Since $Y_{2}$ is reflexive there exists $u_{\beta_{k}} \rightarrow \tilde{u}$ in $L^{p}\left(0, T ; Y_{2}\right)$ from which it follows that $u=\tilde{u} \in L^{p}\left(0, T ; K_{2}\right)$. Since $f^{p}$ is demiclosed the last argument in the proof of Theorem 1.4.1 can be repeated here and the result follows.

From Theorems 1.5.3 and 1.5.4 we have:

THEOREM 1.5.5. - Let the assumptions of Lemma 1.5.2 hold and $g$ be accretive on $K_{1}$. If in addition for each $\left.\left.\beta \in\right] 0,1\right]$ and $v \in L^{\infty}\left(0, T ; K_{2}\right)$ there exists $u_{\beta} \in W^{1, p}\left(0, T ; Y_{1}\right) \cap L^{\infty}\left(0, T ; K_{2}\right)$ satisfying

$$
\left\{\begin{array}{l}
\frac{d u_{\beta}}{d t}+g_{\beta}\left(u_{\beta}\right)=v, \\
u_{\beta}(0)=u_{0},
\end{array} \quad\left\|u_{\beta}\right\|_{2} \leqslant K\left(\|v\|_{2}\right) .\right.
$$

Then for each $w \in L^{p}\left(0, T ; K_{1}\right)$ the problem $(6)$ has a unique strong soluiton $u$. Moreover if $Y_{1}$ and $Y_{2}$ are reflexive, if $|g(u)|_{1}$ is uniformly bounded on bounded subsets of $K_{2}$ in $Y_{2}$ and $w \in L^{\infty}\left(0, T ; K_{2}\right)$ then $u \in W^{1, p}\left(0, T ; Y_{1}\right) \cap L^{p}\left(0, T ; K_{2}\right)(1<p<+\infty)$.

\subsection{Examples.}

In this section we give an example in which the previous theory is applied to partial differential equations. Let $\Omega \subset \boldsymbol{R}^{n}$ be an open bounded domain with smooth boundary and by $H^{m}(\Omega), H_{0}^{m}(\Omega)$ the usual Sobolev spaces. On $\Omega$ we consider the differential operator with real coefficients

$$
E u=\sum_{|p|,|a| \leqslant m}(-1)^{|p|} D^{p}\left(a_{p q} D^{\alpha} u\right)
$$

where $a_{p Q} \in C^{m}(\bar{\Omega})$ and for some positive constant $\gamma$

$$
\sum_{|p|=|q|=m} a_{p q}(x) \xi^{p+q} \geqslant \gamma|\xi|^{2} .
$$

Let $\varphi: \boldsymbol{R} \rightarrow \boldsymbol{R}$, accretive and continuous; we define:

$$
\begin{aligned}
& \left\{\begin{array}{l}
D_{f}=H^{2 m}(\Omega) \cap H_{0}^{m}(\Omega) \\
f u=E u
\end{array}\right. \\
& \left\{\begin{array}{l}
D_{g}=\left\{u \in L^{2}(\Omega), a \varphi \circ u \in L^{2}(\Omega)\right. \\
g(u)=a \varphi \circ u
\end{array}\right.
\end{aligned}
$$

and for each $\lambda \in] 0,1]$

$$
\left\{\begin{array}{l}
D_{g_{\lambda}}=L^{2}(\Omega) \\
g_{\lambda}(u)=a \varphi_{\lambda} \circ u
\end{array}\right.
$$


where $a \in L^{\infty}(\Omega), a \geqslant 0$ a.e. $x \in \Omega^{n}$ and $\varphi_{\lambda}$ are the Yosida's approximating functions of $\varphi$. Furthermore we denote by $X_{1}$ and $X_{2}$ the Hilbert spaces $L^{2}(\Omega)$ and $H^{m}(\Omega)$ with the usual norm \|\|$_{1}$ and \|\|$_{2}$ and suppose $m>n / 2$.

LEMMA 1.6.1. - The following statements hold:

(i) $g_{\lambda}$ is Lipschitz continuous and accretive on $X_{1}$;

(ii) we have $\lim _{\lambda \rightarrow 0^{+}}\left\|g_{\lambda}(u)-g(u)\right\|_{1}=0$ uniformly on bounded subsets of $X_{2}$.

Proof. - (i) follows directly from Lemma 1.2.4. (ii) is a consequence of Sobolev's inequality and the uniform continuity of $\varphi$ on eompact subsets of $\boldsymbol{R}$.

LEmma 1.6.2. - For each $\lambda \in] 0,1]$ and $\mu$ sufficiently large there exists a unique $u_{\lambda} \in D_{f}$ such that

$$
\mu u_{\lambda}+f\left(u_{\lambda}\right)+g_{\lambda}\left(u_{\lambda}\right)=v, \quad v \in X_{1}
$$

and we have

$$
\left\|u_{\lambda}\right\|_{2} \leqslant k\|v\|_{1}^{2}
$$

Proof. - By Garding's inequality there exists $\mu_{0} \geqslant 0$ such that $\mu_{0} I+f$ is $m$-accretive on $X_{1}$. Hence using Theorem 1.2.3 and Lemma 1.6.1 we obtain that (9) has a unique solution $u_{\lambda}$ for $\mu>\mu_{0}$ and we have

$$
\left\|u_{\lambda}\right\|_{1} \leqslant \frac{1}{\mu-\mu_{0}}\|v\|_{1} .
$$

By Garding's inequality there exists $\alpha>0$ such that

$$
\mu\left\|u_{\lambda}\right\|_{1}^{2}+\alpha\left\|u_{\lambda}\right\|_{2}^{2}-\mu_{0}\left\|u_{\lambda}\right\|_{1}^{2}+\left(g_{\lambda}\left(u_{\lambda}\right), u_{\lambda}\right)_{1} \leqslant\|v\|_{1} \cdot\|u\|_{1} .
$$

Combining these results we have

$$
\alpha\left\|u_{\lambda}\right\|_{2}^{2} \leqslant \frac{1}{\mu_{0}-\mu}\|v\|_{1}^{2}
$$

and the proof is complete.

Theorem 1.6.1. - For every $v \in X_{1}$ there exists a unique $u \in D_{f}$ such that

$$
\mu u+f u+g(u)=v, \quad \mu>\mu_{0} .
$$

Proof. - By Theorem 1.3.5 there exists a unique strong solution of (10) and we have $u=\lim _{\lambda \rightarrow 0^{+}} u_{\lambda}$ with $u_{\lambda}$ satisfying (9). Hence by Lemma 1.6.2 (i) and the dominated convergence theorem we obtain $\lim _{\lambda \rightarrow 0^{+}} g_{\lambda}\left(u_{\lambda}\right)=g(u)$ and the conclusion follows.

22 - Annali di Matematica 
From the regularity results for elliptic equations we get the following theorem

THEOREM 1,6.2. - Let $u$ be the solution of $(10)$; if $v \in H^{k}(\Omega)$ and $a \in C^{k}(\bar{\Omega})$ then $u \in H^{2 m+k}(\Omega)$.

Proof. - Let $v \in H^{1}(\Omega), a \in C^{1}(\bar{\Omega})$; by Theorem 1.6.1 there exists $u \in H^{2 m}(\Omega) \cap$ $\cap H_{0}^{m}(\Omega)$ such that $\mu u+f u=v-g(u) \in H^{1}(\Omega)$ so that $u \in H^{2 m+1}(\Omega)$. If $k>1$ the result follows by induction.

\section{PART II}

\section{EVOLUTION EQUATIONS}

\subsection{The continuous case.}

Let $Y_{1}$ be a real Banach space with norm $\left.\right|_{1}$ and $g \in G_{0}\left(Y_{1}\right)$; we will consider the initial value problem

$$
\left\{\begin{array}{l}
\frac{d u}{d t}+g(u)=w \\
u(0)=u_{0}
\end{array} \quad t \in[0, T]\right.
$$

and will obtain criteria for the case in which $g$ is not assumed to be $m$-accretive.

Definition 2.1.1. - Let $w \in C\left(0, T ; Y_{1}\right)$; a function $u:[0, T] \rightarrow Y_{1}$ is called a solution of (1) if

(i) $u(t) \in D_{g}$ for each $t \in[0, T], t \rightarrow g(u(t)) \in C\left(0, T ; Y_{1}\right)$;

(ii) $u \in C^{1}\left(0, T ; Y_{1}\right)$ and satisfies (1).

Let $K_{1} \subset Y_{1}$ a closed convex set containing the origin; for each $r>0, a>0$ we set $K_{1}^{r}=\left\{u \in K_{1},|u|_{1} \leqslant r\right\}, a K_{1}^{*}=\left\{u \in Y_{1},(1 / a) u \in K_{1}\right\}$.

The following theorems contain the main results concerning the case in which $g$ is continuous. The non continuous case will be studied in section 2.5.

THEOREM 2.1.1. - Let $g: K_{1} \rightarrow Y_{1}$ satisfy the following conditions

(i) $g$ is uniformly continuous and bounded on bounded subsets of $K_{1}$;

(ii) for each $r>0$ there exists $\omega(r)$ such that for each $\alpha>0$ and $u, v \in K_{1}^{r}$ we have

$$
|u-v|_{1} \leqslant|u-v+\alpha[\omega(r)(u-v)+g(u)-g(v)]|_{1}
$$

and

$$
(I+\alpha g+\alpha \omega(r) I)\left(a K_{1}^{r}\right) \supset a K_{1}^{r}
$$

for each $a \leqslant 1$. 
In addition, if there exists $b>1$ such that

(iii) $b u_{0} \in K_{1}, b w(t) \in K_{1}$ for each $t \in[0, T]$;

then the initial value problem (1) admits a solution for $T$ sufficiently small. Moreover there exists $\bar{r}$ such that

(iv) $u(t) \in K_{1}^{\bar{r}}$ for each $t \in[0, T]$.

Remark 1. - Assumption (iii) is satisfied if $K_{1}$ is a cone and $u_{0} \in K_{1}, v(t) \in K_{1}$ for each $t \in[0, T]$.

Theorem 2.1.2. - Let the assumptions of Theorem 2.1.1 hold. Then (1) has a unique solution satisfying (iv).

THeoreyr 2.1.3. - In addition to the conditions of Theorem 2.1.1 assume that there exists $\sigma$ such that for each $\alpha>0$ and $u \in K_{1}$ we have

$$
|u|_{1} \leqslant|u+\alpha \sigma u+\alpha g(u)|_{1} .
$$

Then if $K_{1}$ is a cone (1) has a unique solution satisfying (iv) for each $T>0$.

The proof of Theorems 2.1.1, 2.1.2 and 2.1.3 require some preliminaries and will be given later.

\subsection{Accretive functions.}

In this section we recall some known result concerning accretive functions and the subdifferential of the norm which we present without proof (for a proof see e.g. [7]).

Let $X$ be a real Banach space with norm \|\| and let $X^{*}$ be its dual; the value of $x^{*} \in X^{*}$ at $x \in X$ will be denoted by $\left\langle x, x^{*}\right\rangle$.

Defintrion 2.2.1. - With each $x \in X$ we associate the set

$$
\partial\|x\|=\left\{x^{*} \in X^{*}:\|x+y\| \geqslant\|x\|+\left\langle y, x^{*}\right\rangle \text { for each } y \in X\right\}
$$

The function $X \rightarrow 2^{x^{*}}, x \rightarrow \partial\|x\|$ is called the subdifferential of the norm.

The following lemmas hold:

LEMMra 2.2.1. - Conditions (i) and (ii) below on a function $f \in G(X)$ are equivalent:

(i) for each $(x, u) \in f$ and $\alpha>0$ we have

$$
\|x\| \leqslant\|x+\alpha u\|
$$


(ii) there exists $x^{*} \in \partial\|x\|$ such that

$$
\left\langle u, x^{*}\right\rangle \geqslant 0, \quad \text { for each }(x, u) \in f
$$

Lemia 2.2.2. - Conditions (i) and (ii) below on a function $j \in G(X)$ are equivalent:

(i) for every $(x, u),(y, v) \in f$ and $\alpha>0$ we have

$$
\|x-y\| \leqslant\|x-y+\alpha(u-v)\|
$$

(ii) there exists $x^{*} \in \partial\|x-y\|$ such that

$$
\left\langle u-v, x^{*}\right\rangle \geqslant 0, \quad \text { for each }(x, u),(y, v) \in f \text {. }
$$

LEmia 2.2.3. - If $u:[0, T] \rightarrow X$ has the left derivative then $t \rightarrow\|u(t)\|$ has the left derivative and we have:

$$
D_{s}\|u(t)\| \leqslant\left\langle D_{s} u(t), z^{*}\right\rangle, \quad \text { for each } z^{*} \in \partial\|u(t)\| .
$$

\subsection{Proof of Theorems 2.1.1 and 2.1.2.}

The conditions (i) and (ii) of Theorem 2.1.1 are assumed to hold throughout this section.

Set $X_{1}=C\left(0, T ; Y_{1}\right), Q_{1}=C\left(0, T ; K_{1}\right), Q_{1}^{r}=C\left(0, T ; K_{1}^{r}\right)$ and assume that $b u_{0} \in K_{1}$ for some $b>1$. We define

$$
\begin{aligned}
& \left\{\begin{array}{l}
D_{f}=\left\{u \in X_{1}, \frac{d u}{d t} \in X, u(0)=u_{0}\right\}, \\
f(u)=\frac{d u}{d t},
\end{array}\right. \\
& \left\{\begin{array}{l}
D_{\tilde{g}}=Q_{1} \\
(\tilde{g}(u))(t)=\exp \left[-\left(1+\omega_{0}\right) t\right] g\left(u(t) \exp \left[\left(1+\omega_{0}\right) t\right]\right)
\end{array}\right.
\end{aligned}
$$

where $r_{0}>\left|u_{0}\right|_{1} b$ and $\omega_{0}=\omega\left(r_{0}\right)$ is given by Theorem 2.1.1 (i).

Lemina 2.3.1. - If

(i) $\exp \left[\left(1+\omega_{0}\right) T\right]<b$

then $\omega_{0} I+\tilde{g}: Q_{1} \rightarrow X_{1}$ is $m$-accretive on $b^{-1} Q_{1}^{r_{0}}$. 
Proof. - Let $u, v \in b^{-1} Q_{1}^{r_{\sigma}}$; setting

$$
\begin{aligned}
& \tilde{u}(t)=\exp \left[\left(1+\omega_{0}\right) t\right] u(t) \\
& \tilde{v}(t)=\exp \left[\left(1+\omega_{0}\right) t\right] v(t)
\end{aligned}
$$

we have $\tilde{u}(t), \tilde{v}(t) \in Q_{1}^{r_{0}}$ for each $t \in[0, T]$. Using Theorem 2.1.1 we obtain

$$
\begin{aligned}
\mid[u(t)-v(t)]\left(1+\alpha \omega_{0}\right) & +\left.\alpha \exp \left[-\left(1+\omega_{0}\right) t\right][g(\tilde{u}(t))-g(\tilde{v}(t))]\right|_{1}= \\
& =\exp \left[-\left(1+\omega_{0}\right) t\right][\tilde{u}(t)-\tilde{v}(t)]\left(1+\alpha \omega_{0}\right)+\left.\alpha[g(\tilde{u}(t))-g(\tilde{v}(t))]\right|_{1} \geqslant \\
& \geqslant \exp \left[-\left(1+\omega_{0}\right) t\right]\left|\tilde{u}(t)-\tilde{v}(t)_{1}=\right| u(t)-\left.v(t)\right|_{1}
\end{aligned}
$$

from which it follows that $\omega_{0} I+\tilde{g}$ is accretive on $b^{-1} Q_{1}^{\gamma_{0}}$. Now let $v \in b^{-1} Q_{1}^{\gamma_{0}}$; for each $t \in[0, T]$ we have $\tilde{v}(t)=\exp \left[\left(1+\omega_{0}\right) t\right] v(t) \in \exp \left[\left(1+\omega_{0}\right) t\right] b^{-1} K_{1}^{r_{3}}$. Hence using Theorem 2.1.1 (ii) there exists $\tilde{u}(t) \in \exp \left[\left(1+\omega_{0}\right) t\right] b^{-1} K_{1}^{r_{0}}$ such that

$$
\tilde{u}(t)+\alpha \omega_{0} \tilde{u}(t)+\alpha g(\tilde{u}(t))=\tilde{v}(t)
$$

Setting $u(t)=\exp \left[-\left(1+\omega_{0}\right) t\right] \tilde{u}(t)$ we have $u \in b^{-1} Q_{1}^{r_{0}}$ and

$$
u+\alpha \omega_{0} u+\alpha \tilde{g}(u)=v
$$

which yields $\left(I+\alpha \omega_{0} I+\alpha \tilde{g}\right)\left(b^{-1} Q_{v_{0}}^{1}\right) \supset b^{-1} Q_{1}^{r_{a}}$ and the proof is complete.

As a consequence of Lemma 2.3.1 and Theorem 1.2.3 we obtain the following result:

Theorem 2.3.1. - If $\exp \left[\left(1+\omega_{0}\right) T\right]<b$ then for every $v \in b^{-1} Q_{1}^{r_{0}}$ there exists $u \in b^{-1} Q_{1}^{r_{0}} \cap D_{f}$ such that

$$
u+\alpha f(u)+\alpha \omega_{0} u+\alpha \tilde{g}(u)=v .
$$

Proof of Theorem 2.1.1. - Let $r_{0}>\|w\|_{1} b$ and $T$ satisfy Lemma 2.3.1 (i). By Theorem 2.3.1 there exists $\tilde{u}$ satisfying the initial value problem

$$
\left\{\begin{array}{l}
\tilde{u}+\frac{d \tilde{u}}{d t}+\omega_{0} \tilde{u}+\exp \left[-\left(1+\omega_{0}\right) t\right] g\left(\exp \left[\left(1+\omega_{0}\right) t\right] \tilde{u}(t)\right)=\exp \left[-\left(1+\omega_{0}\right) t\right] w(t) \\
\tilde{u}_{0}(0)=u_{0}
\end{array}\right.
$$

and we have $\tilde{u}(t) \in b^{-1} K_{1}^{r_{0}}$ for each $t \in[0, T]$. Hence $u=\exp \left[\left(1+\omega_{0}\right) t\right] \tilde{u}$ satisfies $(1)$ and we have

$$
u(t) \in K_{1}^{r_{\mathrm{n}}}, \quad \text { for each } t \in[0, T] .
$$

Theorem 2.3.1. - Let $u, \bar{u}$ be solutions of (1) with initial datum $u_{0}, \bar{u}_{0}$. If $u(t)$, 
$\bar{u}(t) \in K_{1}^{\bar{p}}$ for each $t \in[0, T]$ then

$$
|u(t)-\bar{u}(t)|_{1} \leqslant \exp [\omega(\bar{r}) t]\left|u_{0}-u_{0}\right|_{1} .
$$

Proof. - By assumption we have

$$
\frac{d}{d t}(u-\bar{u})+g(u)-g(\bar{u})=0, \quad u(0)-\bar{u}(0)=u_{0}-\bar{u}_{0} .
$$

By Lemma 2.2.2 and Theorem 2.1.1 (ii) there exists $z^{*}(t) \in \partial|u(t)-\bar{u}(t)|_{1}$ such that

$$
\begin{aligned}
\left\langle\frac{d}{d t}(u-\bar{u}), z^{*}(t)\right\rangle_{1}-\omega(r) \mid u(t)- & \left.\bar{u}(t)\right|_{1} \leqslant \\
& \leqslant\left\langle\frac{d}{d t}(u-\bar{u}), z^{*}(t)\right\rangle_{1}+\left\langle g(u(t))-g(\bar{u}(t)), z^{*}(t)\right\rangle_{1}=0 .
\end{aligned}
$$

Hence using Lemma 2.2.3

$$
D_{s}|u(t)-\bar{u}(t)|_{1}-\omega(r)|u(t)-\bar{u}(t)|_{1} \leqslant 0
$$

and the result follows.

Proof of Theorem 2.1.2. - It follows directly from Theorem 2.3.1 by assuming $u_{0}=\bar{u}_{0}$.

\subsection{Proof of Theorem 2.1.3.}

LEMMA 2.4.1. - Let $\bar{u}$ be a solution of problem (1); if there exists $\sigma$ such that

$$
|u|_{1} \leqslant|u+\alpha \sigma u+\alpha g(u)|_{1}
$$

for each $u \in K_{1}$ then

for each $t \in[0, T]$.

$$
|\bar{u}(t)|_{1} \leqslant \exp [\sigma t]\left|u_{0}\right|_{1}+\int_{0}^{t} \exp [\sigma(t-s)]|w(s)|_{1} d s
$$

Proof. - By Lemma 2.2.1 there exists $z^{*} \in \partial|u(t)|_{1}$ such that

$$
\left\langle\frac{d \bar{u}}{d t}, z^{*}(t)\right\rangle_{1}-\sigma|u(t)|_{1} \leqslant\left\langle\frac{d \bar{u}}{d t}, z^{*}(t)\right\rangle_{1}+\left\langle g(\bar{u}(t)), z^{*}(t)\right\rangle_{1} \leqslant|w(t)|_{1}
$$

Hence using Lemma 2.2.3

$$
D_{s}|u(t)|_{1}-\sigma|u(t)|_{1} \leqslant|w(t)|_{1}
$$

and the result follows. 
Proof of THEOREM 2.1.3. - Let $T^{*}$ be supremum of the set of all $T$ such that (1) has a unique solution and let $u$ be such that

$$
\left\{\begin{array} { l } 
{ \frac { d u } { d t } + g ( u ) = w , } \\
{ u ( 0 ) = u _ { 0 } }
\end{array} \quad t \in \left[0, T^{*}[.\right.\right.
$$

We have $(s<t)$

$$
|u(t)-u(s)|_{1} \leqslant \int_{s}^{t}|g(u(\tau))|_{1} d \tau+\int_{s}^{t}|w(\tau)|_{1} d \tau \rightarrow 0
$$

where we used Lemma 2.4.1 and assumption (i) of Theorem 2.1.1. Let $T^{*}<+\infty$; setting $u\left(T^{*}\right)=\lim _{t \rightarrow T^{*}} u(t)$ we have

$$
\lim _{t \rightarrow T^{*}}\left|g(u(t))-g\left(u\left(T^{*}\right)\right)\right|_{1}=0
$$

from which it follows that $T^{*}$ is a maximum so, using standard arguments, that $T^{*}=+\infty$. Since the uniqueness follows by Theorem 2.3.1 the proof is complete.

\subsection{The noncontinuous case.}

We are interested in the solution of the initial value problem (1) if $g$ is not supposed to be continuous. To state our results more precisely it is convenient to introduce some notations and definitions.

Definition 2.5.1. - Let $w \in X_{1}$; a function $u \in X_{1}$ is called a strong solution of (1) if there exists $\left\{u_{\beta}\right\}$ such that

$$
\begin{aligned}
& \text { (i) } u_{\beta} \in O^{1}\left(0, T ; Y_{1}\right), u_{\beta}(0)=u_{0}, \lim _{\beta \rightarrow 0^{+}} u_{\beta}=u \text { in } X_{1} \text {, } \\
& \text { (ii) } u_{\beta}(t) \in D_{g} \text { for each } t \in[0, T], t \rightarrow g\left(u_{\beta}(t)\right) \in X_{1}, \\
& \text { (iii) we have } \lim _{\beta \rightarrow 0^{+}} d u_{\beta} / d t+g\left(u_{\beta}\right)=w \text { in } X_{1} .
\end{aligned}
$$

Let $Y_{2} \subset Y_{1}$ be a real Banach space with norm ||$_{2}$ and $K_{2}=K_{1} \cap Y_{2}$. For each $r>0, a>0$ we set

$$
K_{2}^{r}=\left\{u \in K_{2},|u|_{2} \leqslant r\right\}, \quad a K_{2}^{r}=\left\{u \in Y_{2}: \frac{1}{a} u \in K_{2}^{r}\right\}
$$

and suppose that $Y_{1}$ and $Y_{2}$ have the property that $K_{2}$ is closed in $Y_{1}$.

The following theorems contain the main results concerning the non continuous case : 
Theorem 2.5.1. - Let $D_{g} \supset K_{2}$ and assume that there exists $\left\{g_{\beta}\right\}$ such that

(i) $g_{\beta}$ is uniformly continuous and bounded on bounded subsets of $K_{1}$;

(ii) for each $r>0$ there exists $\omega(r)$ such that $\omega(r) I+g_{\beta}$ is accretive on $K_{2}^{r}$ and we have

$$
\left(I+\alpha \omega(r) I+\alpha g_{\beta}\right)\left(a K_{2}^{r}\right) \supset a K_{2}^{r}, \quad \alpha>0
$$

for each $a \leqslant 1$;

(iii) we have $\lim _{\beta \rightarrow 0}\left|g_{\beta}(u)-g(u)\right|_{1}=0$ uniformly on bounded subsets of $K_{2}$ in $Y_{2}$.

In addition, if $u_{0} \in K_{2}, w(t) \in K_{2}$ for each $t \in[0, T]$ and there exists $b>1$ such that

(iv) $b u_{0} \in K_{1}, b w(t) \in K_{1}$ for each $t \in[0, T]$;

then for $T$ sufficiently small the initial value problem (1) admits a strong solution $u$; moreover there exists $\bar{r}$ such that

(v) $u(t) \in \bar{K}_{2}^{\bar{r}}$ for each $t \in\left[0, x^{\prime}\right]$.

Theorem 2.5.2, - Let the assumptions of Theorem 2.5.1 hold. Then (1) has a unique strong solution $u$ such that $u$ is limit of functions satisfying $(\mathrm{v})$.

THEOREM 2.5.3. - Let the assumptions of Theorem 2.5.1 hold. If $K_{1}$ is a cone and there exists $\sigma$ such that

$$
|u|_{2} \leqslant\left|u+\alpha \sigma u+\alpha g_{\beta}(u)\right|_{2}, \quad \alpha>0
$$

for each $u \in K_{2}$ then for each $T>0$ the problem (1) has a unique strong solution satisfying $(v)$.

REMARK 2. - The conclusion of Theorem 2.5.3 can be established without requiring that $K_{2}$ is closed in $Y_{1}$.

\subsection{Proof of Theorems 2.5.1 and 2.5.2.}

The assumptions of Theorem 2.5.1 are assumed to hold throughout this section.

Set $Q_{2}=C\left(0, T ; K_{2}\right), Q_{2}^{r}=C\left(0, T ; K_{2}^{r}\right)$ and assume that $b u_{0} \in K_{1}$ for some $b>1$. We define

$$
\begin{aligned}
& \left\{\begin{array}{l}
D_{\tilde{o}_{\beta}}=Q_{1} \\
\left(\tilde{g}_{\beta}(u)\right)(t)=\exp \left[-\left(1+\omega_{0}\right) t\right] g_{\beta}\left(\exp \left[\left(1+\omega_{0}\right) t\right] u(t)\right)
\end{array}\right. \\
& \left\{\begin{array}{c}
D_{\tilde{g}}=\left\{u \in X_{1}: \exp \left[\left(1+\omega_{0}\right) t\right] u(t) \in D_{v}\right. \\
\left.\quad \text { for each } t \in[0, T], t \rightarrow g\left(\exp \left[\left(1+\omega_{0}\right) t\right] u(t)\right) \in X_{1}\right\} \\
(\tilde{g}(u))(t)=\exp \left[-\left(1+\omega_{0}\right) t\right] g\left(\exp \left[\left(1+\omega_{0}\right) t\right] u(t)\right)
\end{array}\right.
\end{aligned}
$$

where $r_{0}>\left|u_{0}\right|_{2} b$ and $\omega_{0}=\omega\left(r_{0}\right)$ is defined as in (ii) of Theorem 2.5.1. 
We have

LEMMA 2.6.1. - If $\exp \left[\left(1+\omega_{0}\right) T\right]<b$ and $D_{g} \supset K_{2}$ then for each $r>0, D_{\tilde{g}} \supset b^{-1} Q_{1} \cap$ $\cap Q_{2}^{r}$ and we have

(i) $\lim _{\beta \rightarrow 0^{+}}\left\|\tilde{g}(u)-\tilde{g}_{\beta}(u)\right\|_{1}=0$

uniformly in $b^{-1} Q_{1} \cap Q_{2}^{r}$.

Proof. - If $u \in b^{-1} Q_{1}$ then $\exp \left[\left(1+\omega_{0}\right) t\right] u(t) \in K_{1}$ for each $t \in[0, T]$ so that $u \in$ $\in b^{-1} Q_{1} \cap Q_{2}^{r}$ yields $u(t) \exp \left[\left(1+\omega_{0}\right) t\right] \in K_{2}^{r b} \cap D_{g}$ for each $t \in[0, T]$. Now using assumption (iv) of Theorem 2.5.1 we obtain

$$
\lim _{\beta \rightarrow 0^{+}}\left|g_{\rho}\left(\exp \left[\left(1+\omega_{0}\right) t\right] u(t)\right)-g\left(\exp \left[\left(1+\omega_{0}\right) t\right] u(t)\right)\right|_{1}=0
$$

uniformly in $t$ on $b^{-1} Q_{1} \cap Q_{2}^{r}$. This implies that $t \rightarrow g\left(\exp \left[\left(1+\omega_{0}\right) t\right] u(t)\right) \in X_{1}$ and (i).

The existence Theorem 2.3.1 can be extended to the functions $g_{\beta}$ with unessential changes and we obtain the following result:

Lemad 2.6.2. - If $\exp \left[\left(1+\omega_{0}\right) T\right]<b$ then for every $v \in b^{-1} Q_{2}^{r_{0}}$ there exists $u_{\beta} \in$ $\in b^{-1} Q_{2}^{r_{0}} \cap D_{f}$ such that

$$
u_{\beta}+\alpha f\left(u_{\beta}\right)+\alpha \omega_{0} u_{\beta}+\alpha \tilde{g}_{\beta}\left(u_{\beta}\right)=v .
$$

Theorem 2.6.1. - If $\exp \left[\left(1+\omega_{0}\right) T\right]<b$ and $D_{g} \supset K_{2}$ then for each $v \in b^{-1} Q_{2}^{r_{0}}$ there exists $u \in b^{-1} Q_{2}^{r_{0}}$ strong solution of the equation

$$
u+\alpha \omega_{0} u+\alpha f(u)+\alpha \tilde{g}(u)=v
$$

and we have $u=\lim _{\beta \rightarrow 0^{+}} u_{\beta}$ with $u_{\beta}$ defined as in Lemma 2.6.2.

Proof. - Let $u_{\beta}$ be defined as in Lemma 2.6.2; we have

$$
u_{\beta}+\alpha \omega_{0} u_{\beta}+\alpha f\left(u_{\beta}\right)+\alpha \tilde{g}\left(u_{\beta}\right)=v+\alpha\left[\tilde{g}\left(u_{\beta}\right)-\tilde{g}_{\beta}\left(u_{\beta}\right)\right]
$$

and

$$
u_{\beta}-u_{\gamma}+\alpha\left[f\left(u_{\beta}\right)-f\left(u_{\gamma}\right)\right]+\alpha\left[\omega_{0}\left(u_{\beta}-u_{\gamma}\right)+\tilde{g}_{\beta}\left(u_{\beta}\right)-\tilde{g}_{\beta}\left(u_{\gamma}\right)\right]=\alpha\left[\tilde{g}_{\gamma}\left(u_{\gamma}\right)-g_{\beta}\left(u_{\gamma}\right)\right]
$$

hence by the accretiveness of $f+\tilde{g}_{\beta}$

$$
\left\|u_{\beta}-u_{\eta}\right\|_{1} \leqslant \alpha\left\|\tilde{g}_{\nu}\left(u_{\gamma}\right)-\tilde{g}_{\beta}\left(u_{\gamma}\right)\right\|_{1}
$$

In view of Lemma 2.6.1 the proof is complete. 
Proof of THEOREM 2.5.1. - Let $r_{0}>\|w\|_{2} b$ and $\exp \left[\left(1+\omega_{0}\right) T\right]<b$. By Theorem 2.6.1 there exists $\tilde{u} \in b^{-1} Q_{2}^{r_{0}}$ strong solution of the Cauchy problem

$$
\left\{\begin{array}{l}
\tilde{u}+\frac{d \tilde{u}}{d t}+\omega_{0} \tilde{u}+\exp \left[-\left(1+\omega_{0}\right) t\right] g\left(\exp \left[\left(1+\omega_{0}\right) t\right] \tilde{u}(t)\right)=\exp \left[-\left(1+\omega_{0}\right) t\right] w(t) \\
\tilde{u}(0)=u_{0}
\end{array}\right.
$$

Hence $u=\exp \left[\left(1+\omega_{0}\right) t\right] \tilde{u}$ is strong solution of problem (1) and we have $u(t) \in K_{2}^{r_{0}}$ for each $t \in[0, T]$.

THEOREM 2.6.2. - Let $u=\lim _{\beta \rightarrow 0^{+}} u_{\beta}$ and $\bar{u}=\lim _{\beta \rightarrow 0^{+}} \bar{u}_{\beta}$ strong solutions of (1) with initial datum $u_{0}, \bar{u}_{0}$. If there exists $r>0$ such that $u_{\beta}(t), \bar{u}_{\beta}(t) \in K_{2}^{r}$ for each $t \in[0, T]$ then

$$
|u(t)-\bar{u}(t)|_{1} \leqslant \exp [\omega(r) t]\left|u_{0}-\bar{u}_{0}\right|_{1} .
$$

Proof. - By assumption we have

$$
\begin{aligned}
& \lim _{\beta \rightarrow 0^{+}} u_{\beta}=u, \quad \lim _{\beta \rightarrow 0^{+}} \bar{u}_{\beta}=\bar{u}, \\
& u_{\beta}(0)=u_{0}, \quad \bar{u}_{\beta}(0)=\bar{u}_{0}, \\
& \lim _{\beta \rightarrow 0^{+}} \frac{d}{d t} u_{\beta}+g\left(u_{\beta}\right)=\lim _{\beta \rightarrow 0^{+}} \frac{d}{d t} \bar{u}_{\beta}+g\left(\bar{u}_{\beta}\right)=w,
\end{aligned}
$$

so that

$$
\begin{aligned}
& \frac{d}{d t}\left(u_{\beta}-\bar{u}_{\beta}\right)+g\left(u_{\beta}\right)-g\left(\bar{u}_{\beta}\right)=w_{\beta} \rightarrow 0, \\
& u_{\beta}(0)-\bar{u}_{\beta}(0)=u_{0}-\bar{u}_{0} .
\end{aligned}
$$

The arguments in the proof of Theorem 2.3.1 can be repeated here in an obvious way and the result follows.

Proof of THEorem 2.5.2. - It follows directly from Theorem 2.6 .2 by assuming $u_{0}=\bar{u}_{0}$.

REMARK 3. - Let $u$ be strong solution of problem (1); it is easy to verify that if the hypotheses of Theorem 2.5.1 hold then

$$
u(t)=u_{0}+\int_{0}^{t}[-g(u(s))+w(s)] d s
$$

for each $t \in[0, T]$. 


\section{7. - Proof of Theorem 2.5.3.}

Lemia 2.7.1. - Let $u_{\beta}$ be a solution of the Canehy problem

$$
\left\{\begin{array}{l}
\frac{d u_{\beta}}{d t}+g_{\beta}\left(u_{\beta}\right)=w \\
u_{\beta}(0)=u_{0}
\end{array} \quad t \in[0, T], u_{0} \in K_{2}, w \in Q_{2} .\right.
$$

If there exists $\sigma$ such that $|u|_{2} \leqslant\left|u+\alpha \sigma u+\alpha g_{\beta}(u)\right|_{2}$ for each $u \in K_{2}$ then

$$
\left|u_{\beta}(t)\right|_{2} \leqslant \exp [\sigma t]\left|u_{0}\right|_{2}+\int_{0}^{t} \exp [\sigma(t-s)]|w(s)|_{2} d s
$$

for each $t \in[0, T]$.

Proof. - By Lemma 2.2.1 ther exists $z^{*}(t) \in \partial\left|u_{\beta}(t)\right|_{2}$ such that

$$
\left\langle g_{\beta}\left(u_{\beta}(t)\right), z^{*}(t)\right\rangle \geqslant-\sigma\left|u_{\beta}(t)\right|_{2}
$$

so Lemma 2.2.3 yields

$$
D_{\mathrm{s}}\left|u_{\beta}(t)\right|_{2}-\sigma\left|u_{\beta}(t)\right|_{2} \leqslant|w(t)|_{2}
$$

and the result follows.

Lemma 2.7.2. - Let the hypotheses of Theorem 2.5.3 hold; then the initial value problem (2) has a unique solution $u_{\beta}$ for each $T>0$.

Proof. - This follows from Lemma 2.7.1 by using the same arguments as in the proof of Theorem 2.1.3.

Proof of Theorem 2.5.3. - Let $u_{\beta}$ be defined as in Lemma 2.7.1; we have:

$$
\frac{d}{d t}\left(u_{\beta}-u_{\gamma}\right)+g_{\beta}\left(u_{\beta}\right)-g_{\beta}\left(u_{\gamma}\right)=g_{\nu}\left(u_{\gamma}\right)-g_{\beta}\left(u_{\gamma}\right) .
$$

By Lemma 2.7.1 there exists $r$ such that $\left|u_{\beta}(t)\right|_{2} \leqslant r$ for each, $t \in[0, T]$ hence, using assumption (ii) of Theorem 2.5.1 and Lemma 2.2.2, we obtain

$$
\begin{aligned}
\left\langle\frac{d}{d t}\left(u_{\beta}-u_{\gamma}\right), z^{*}(t)\right\rangle_{1}-\omega(r) \mid u_{\beta}(t) & -u_{\gamma}(t)_{1} \mid \leqslant\left\langle\frac{d}{d t}\left(u_{\beta}-u_{\gamma}\right), z^{*}(t)\right\rangle_{1}+ \\
& +\left\langle g_{\beta}\left(u_{\beta}(t)\right)-g_{\beta}\left(u_{\gamma}(t)\right), z^{*}(t)\right\rangle_{1} \leqslant\left|g_{\gamma}\left(u_{\gamma}(t)\right)-g_{\beta}\left(u_{\gamma}(t)\right)\right|_{1} .
\end{aligned}
$$


Now Lemma 2.2.3 gives

$$
D_{s}\left|u_{\beta}(t)-u_{\gamma}(t)\right|_{1}-\omega(r)\left|u_{\beta}(t)-u_{\gamma}(t)\right|_{1} \leqslant\left|g_{\gamma}\left(u_{\gamma}(t)\right)-g_{\beta}\left(u_{\gamma}(t)\right)\right|_{1}
$$

from which it follows that

$$
\left|u_{\beta}(t)-u_{\gamma}(t)\right|_{1} \leqslant \int_{0}^{t} \exp [\omega(r)(t-s)]\left|g_{\gamma}\left(u_{\gamma}(s)\right)-g_{\rho}\left(u_{\gamma}(s)\right)\right|_{1} d s .
$$

In view of Lemma 2.7.1 and assumption (iv) of Theorem 2.5.1 there exists $u \in X_{1}$ such that $\lim _{\beta \rightarrow 0^{+}} u_{\beta}=u$ and we have

$$
\lim _{\beta \rightarrow 0^{+}}\left(\frac{d u_{\beta}}{d t}+g\left(u_{\beta}\right)\right)=\lim _{\beta \rightarrow 0^{+}}\left(w+g\left(u_{\beta}\right)-g_{\beta}\left(u_{\beta}\right)\right)=w .
$$

Since the uniqueness follows from Theorem 2.6.2 the proof is complete.

\section{8. - Local and global regularity.}

In this section we will give conditions guaranteeing the differentiability of the strong solution of the problem (1).

As a first result we have:

Theorem 2.8.1. - Let $Y_{0} \hookrightarrow Y_{1} \hookrightarrow Y_{2}, K_{0} \subset Y_{0}$ a closed convex set containing the origin and $K_{1}=K_{0} \cap Y_{1}, K_{2}=K_{0} \cap Y_{2}$. Let $g \in G_{0}\left(Y_{0}\right)$ such that $D_{g} \supset K_{1}$ and assume that there exists $\left\{g_{\beta}\right\} \subset G_{0}\left(Y_{\theta}\right)$ such that

(i) $g_{\beta}$ is uniformly bounded and continuous on bounded subsets of $K_{1}$;

(ii) for each $r>0$ there exists $\omega(r)$ such that for each $\alpha>0$ and $u, v \in K_{2}^{r}$ we have

$$
|u-v|_{1} \leqslant\left|u-v+\alpha\left[\omega(r)(u-v)+g_{\beta}(u)-g_{\beta}(v)\right]\right|_{1}
$$

(iii) for each $r>0$ there exists $\sigma(r) \leqslant \omega(r)$ such that for each $u \in K_{2}^{r}$ and $a<1$ we have

$$
\begin{aligned}
& |u|_{2} \leqslant\left|u+\alpha \sigma(r) u+\alpha g_{\beta}(u)\right|_{2} \\
& \left(I+\alpha \omega(r) I+\alpha g_{\beta}\right)\left(a K_{2}^{r}\right) \supset a K_{2}^{r}
\end{aligned}
$$

(iv) $\lim \left|g_{\beta}(u)-g(u)\right|_{1}=0$ uniformly on bounded subsets of $K_{2}$ in $Y_{2}$;

(v) if $u, u_{\beta} \in K_{1}$ and $\lim _{\beta \rightarrow 0^{+}}\left|u_{\beta}-u\right|_{1}=0$ then $\lim _{\beta \rightarrow 0^{+}}\left|g\left(u_{\beta}\right)-g(u)\right|_{0}=0$.

Assume in addition that $u_{0} \in K_{2}, w(t) \in K_{2}$ for each $t \in[0, T]$ and that there ex- 
ists $b>1$ such that $b u_{0} \in K_{1}$ and $b w(t) \in K_{1}$ for each $t \in[0, T]$; then if $T$ is sufficiently small the initial value problem (1) has a unique solution $u \in C^{1}\left(0, T ; Y_{0}\right) \cap$ $\cap C\left(0, T ; K_{2}\right)$.

Proof. - By Theorem 2.5.1 there exists $u=\lim u_{\beta}$ in $C\left(0, T ; Y_{1}\right)$ strong solution of problem (1). Using $(\nabla)$ we have

$$
\lim \frac{d u_{\beta}}{d t}=\lim \left[-g\left(u_{\beta}\right)+w\right]=-g(u)+w
$$

in $C\left(0, T ; Y_{0}\right)$ which implies $u \in C^{1}\left(0, T ; Y_{0}\right)$ and $d u / d t+g(u)=w$.

The following theorem can be shown in the same way:

ThEorem 2.8.2. - Let the assumptions of Theorem 2.8.1 hold; if $\sigma(r)$ defined as in (iii) ean be taken independent of $r$ then the problem (1) has a unique solution $u \in C^{1}\left(0, T ; Y_{0}\right) \cap C\left(0, T ; K_{2}\right)$ for each $T>0$.

\section{9. - Linear perturbations.}

Let $A$ be a linear operator. In this section we shall consider the initial value problem:

$$
\left\{\begin{array}{l}
\frac{d u}{d t}+A u+g(u)=w \\
u(0)=u_{0}
\end{array} \quad t \in[0, T]\right.
$$

and give sufficient conditions assuring the existence of a solution in some generalized sense for problem (3); more precisely we shall investigate the existence of function $u$ strong solution of the equation

$$
(\overline{f+A})(u)+\tilde{g}(u)=w
$$

where $f$ is defined as in section 2.3 and

$$
\left\{\begin{array}{l}
D_{\tilde{g}}=\left\{u \in X_{1}, u(t) \in D_{g} \text { for each } t \in[0, T], t \rightarrow g(u(t)) \in X_{1}\right\} \\
(\tilde{g}(u))(t)=g(u(t)) .
\end{array}\right.
$$

Replacing $f$ by $\overline{f+A}+e I$ in the proof of Theorems 2.5.1, 2.5.2, 2.5.3, 2.8.1 and 2.8.2 we obtain the following results:

THEOREM 2.9.1. - In addition to the conditions of Theorem 2.5.1 suppose that

(i) $-A+c I$ generates a linear contraction semigroup which leaves $K_{1}$ invariant for some $e \in R$ 
(ii) for each $u \in D_{A}$ we have

$$
|u|_{2} \leqslant|u+\alpha c u+\alpha A u|_{2} .
$$

Then if $u_{0} \in D_{A}$ and $T$ is sufficiently small the initial value problem (3) has a unique solution $u$ in the generalized sense introduced above such that $u$ is limit of functions satisfying condition (vi) of Theorem 2.5.1.

ThEorem 2.9.2. - Let the assumptions of Theorems 2.5.3 and 2.9.1 hold. Then for each $T>0$ the Cauchy problem (3) has a unique solution defined as in Theorem 2.9.1.

Theorem 2.9.3. - For $A \in G_{0}\left(Y_{0}\right)$ define

$$
\left\{\begin{array}{l}
D_{A_{1}}=\left\{u \in Y_{1}, A u \in Y_{1}\right\}, \\
A_{\mathbf{1}} u=A u
\end{array}\right.
$$

In addition to the conditions of Theorem 2.8.1 suppose that $u_{0} \in D_{A_{1}}$ and that $A_{1}$ satisfies assumptions (i) and (ii) of Theorem 2.9.1. Then if $T$ is sufficiently small there is a unique $u$ such that there exists $\left\{u_{\beta}\right\}$ verifying

(i) $u_{\beta} \in C^{1}\left(0, T ; Y_{0}\right) \cap C\left(0, T ; K_{2}\right), u_{\beta}(t) \in D_{A_{1}}$ for each $t \in[0, T]$,

(ii) $u_{\beta}(0)=u_{0}, \lim _{\beta \rightarrow 0^{+}} u_{\beta}=u$ in $C\left(0, T ; Y_{1}\right)$,

(iii) $\lim _{\beta \rightarrow 0^{+}}\left[d u_{\beta} / d t+A u_{\beta}\right]=-g(u)+w$ in $C\left(0, T ; Y_{0}\right)$.

Theorean 2.9.4. - Let the assumptions of Theorems 2.8.2 and 2.9.3 hold. Then for each $T>0$ there exists $u$ defined as in Theorem 2.9.3.

\subsection{0. - Examples.}

Let $Y_{i}$ be the Banach space of measurable functions $u: R^{n} \rightarrow R$ such that

$$
|u|_{i}=\int\left(1+|x|^{2}\right)^{i-1}|u| d x<+\infty, \quad i=1,2
$$

equipped with the norm $\mid \|_{i}$. We consider the integral operator $(u, v) \rightarrow g^{+}(u, v)-$ $-g-(u, v)$ defined as follows

$$
\begin{aligned}
& g^{+}(u, v)=\frac{1}{2}\left[u(x) \int a(y) v(y) d y+v(x) \int a(y) u(y) d y\right] \\
& g^{-}(u, v)=\frac{1}{2}\left[a(x) u(x) \int v(y) d y+a(x) v(x) \int u(y) d y\right]
\end{aligned}
$$


where $a(x)=\sqrt{1+|x|^{2}}$, and for each $\left.\left.\beta \in\right] 0,1\right]$

$$
\begin{aligned}
& g_{\beta}^{+}(u, v)=\frac{1}{2}\left[u(x) \int a_{\beta}(y) v(y) d y+v(x) \int a_{\beta}(y) u(y) d y\right] \\
& g_{\beta}^{+}(u, v)=\frac{1}{2}\left[a_{\beta}(x) u(x) \int v(y) d y+a_{\beta}(x) v(x) \int u(y) d y\right]
\end{aligned}
$$

where $a_{\beta}(x)=(1+\beta a(x))^{-1} a(x)$. Finally we denote by $K_{1}$ the closed convex set $K_{1}=\left\{u \in Y_{1},|u|_{1} \leqslant 1\right\}$ and define

$$
\begin{aligned}
& \left\{\begin{array}{l}
D_{g}=\left\{u \in Y_{1}, g(u, u) \in Y_{1}\right\}, \\
g(u)=g(u, u),
\end{array}\right. \\
& \left\{\begin{array}{l}
D_{o_{\beta}}=Y_{1}, \\
g_{\beta}(u)=g_{\beta}(u, u) .
\end{array}\right.
\end{aligned}
$$

LEMMA 2.10.1. - The following statements hold:

(i) $2 a_{\beta} I+g_{\beta}$ is Lipschitz continuous on bounded subsets of $Y_{1}$;

(ii) if $u, v \in K_{2}^{r}$ then

$$
|u-v|_{1} \leqslant\left|u-v+2 r \alpha(u-v)+2 \alpha a_{\beta}(u-v)+\alpha\left(g_{\beta}(u)-g_{\beta}(v)\right)\right|_{1}
$$

(iii) if $u \in K_{2}^{r}$ and $a<1$ then

$$
\begin{aligned}
& |u|_{2} \leqslant\left|u+2 r \alpha u+2 \alpha a_{\beta} u+\alpha g_{\beta}(u)\right|_{2} \\
& \left(I+2 r \alpha I+\alpha 2 a_{\beta} I+\alpha g_{\beta}\right)\left(a K_{2}^{r}\right) \supset a K_{2}^{r}
\end{aligned}
$$

(iv) we have $D_{g} \supset K_{2}$ and $\lim \left|a_{\beta} u+g_{\beta}(u)-a u-g(u)\right|_{1}=0$ uniformly on bounded subsets of $Y_{2}$.

Proof. - Assertion (i) is evident. Assertion (ii) follows from Lemma 2.2.2 by noting that $\operatorname{sgn}(u-v) \in \partial|u-v|_{ \pm}\left({ }^{3}\right)$ and that if $u, v \in K_{2}^{r}$ we have

$$
\begin{aligned}
& \left\langle 2 a_{\beta}(u-v)+g_{\beta}(u)-g_{\beta}(v), \operatorname{sgn}(u-v)\right\rangle_{1}=2 \int a_{\beta}|u-v| d x+ \\
& \quad+\frac{1}{2}\left[\int|u-v| d x \int a_{\beta}(u+v) d y+\int(u+v) \operatorname{sgn}(u-v) d x \int a_{\beta}(u-v) d y\right] \\
& \quad-\frac{1}{2}\left[\int a_{\beta}|u-v| d x \int(u+v) d y+\int a_{\beta}(u+v) \operatorname{sgn}(u-v) d x \int(u-v) d y\right] \geqslant \\
& \quad \geqslant 2 \int a_{\beta}|u-v| d x-\int|u+v| d x \int a_{\beta}|u-v| d y-\int|u-v| d x \int a_{\beta}|u+v| d y \geqslant-2 r|u-v|_{1} .
\end{aligned}
$$

(3) $\operatorname{sgn} u$ is the function defined as follows

$$
\operatorname{sgn} u \begin{cases}=1 & \text { if } u>0 \\ =0 & \text { if } u=0 \\ =-1 & \text { if } u<0\end{cases}
$$


To prove (iii) take $v \in \mathcal{K}_{2}^{r}$ and define

$$
\begin{aligned}
& u_{1}=v \\
& \ldots \ldots \ldots \\
& u_{n}=\frac{1}{1+\alpha \alpha_{\beta}} \frac{v-g_{\beta}^{+}\left(u_{n-1}\right)}{1+\left(2 r \alpha /\left(1+\alpha a_{\beta}\right)\right)+\alpha\left(\left(\alpha_{\beta}-\left(u_{n-1}\right)^{-1} g_{\bar{\beta}}^{-}\left(u_{n-1}\right)\right) /\left(1+\alpha a_{\beta}\right)\right)} .
\end{aligned}
$$

We have

$$
\begin{aligned}
& \left|u_{1}\right|_{1}=|v| \leqslant 1, \quad\left|u_{1}\right|_{2}=|v|_{2} \leqslant r^{2} \\
& \ldots \ldots \ldots \ldots, \quad \ldots \ldots \ldots \ldots \\
& \left|u_{n}\right|_{1} \leqslant \frac{1}{1+\alpha 2 r}\left(|v|_{1}+\alpha\left|u_{n-1}\right|_{1}\left|u_{n-1}\right|_{2}\right) \leqslant \frac{1+\alpha r}{1+\alpha 2 r} \leqslant 1, \\
& \left|u_{n}\right|_{2} \leqslant \frac{1}{1+\alpha 2 r}\left(|v|_{2}+\alpha\left|u_{n-1}\right|_{2}^{2}\right) \leqslant \frac{r+\alpha r^{2}}{1+\alpha 2 r} \leqslant r .
\end{aligned}
$$

In view of (i) there exists $\lim u_{n}=u \in K_{2}^{r}$ and we have

$$
u+2 r \alpha u+\alpha 2 a_{\beta} u+\alpha g_{\beta}(u)=v
$$

so that

$$
\left(I+\alpha 2 r I+\alpha 2 a_{\beta} I+\alpha g_{\beta}\right)\left(K_{2}^{r}\right) \supset K_{2}^{r} .
$$

Now let $u \in K_{2}^{*}$; we have

$$
\begin{aligned}
\left\langle 2 a_{\beta} u+g_{\beta}(u), \operatorname{sgn} u\right\rangle_{2}=\int 2 a_{\beta}|u|\left(1+|x|^{2}\right) d x+ & \\
& +\int|u|\left(1+|x|^{2}\right) d x \int u a_{\beta} d y-\int a_{\beta}|u|\left(1+|x|^{2}\right) d x \int u d y \geqslant-r|u|_{2}
\end{aligned}
$$

hence combining these results and using Lemma 2.2.1 the conclusion follows. Finally we show (iv); if $u \in K_{2}^{r}$ then

$$
\left|a_{\beta} u+g_{\beta}(u)-a u-g(u)\right|_{1} \leqslant \beta|u|_{2}+2 \beta|u|_{1}|u|_{2}
$$

and the result follows.

As a consequence of Lemma 2.10.1, and Theorem 2.5.1 we have

TheOREM 2.10.1. - Assume that $\left|u_{0}\right|_{1}<1$ and $u_{0} \in Y_{2}$. Then if $T$ is sufficiently small for every $w \in C\left(0, T ; K_{2}\right)$ such that sup $|w(t)|_{1}<1$ the initial value problem

$$
\left\{\begin{array}{l}
\frac{d u}{d t}+a u+g(u)=w \\
u(0)=u_{0}
\end{array}\right.
$$


has a unique strong solution $u$ such that $u$ is limit of functions of $C\left(0, T ; K_{2}^{r}\right)$ for some $r>0$.

Now set $\widetilde{K}_{1}=\left\{u \in Y_{1},|u|_{1} \leqslant 1, u \geqslant 0\right.$ a.e. $\left.x \in R^{n}\right\}$; we have:

LEMma 2.10.2. - The following statements hold:

(i) $2 a_{\beta} I+g_{\beta}$ is Lipschitz continuous on bounded subsets of $Y_{1}$;

(ii) if $u, v \in \bar{K}_{2}^{r}$ then

$$
|u-v|_{1} \leqslant\left|u-v+\alpha a_{\beta}(u-v)+\alpha\left[g_{\beta}(u)-g_{\beta}(v)\right]\right|_{1}
$$

(iii) if $a<1$ and $u \in K_{2}^{r}$ then

$$
\begin{aligned}
& |u|_{2} \leqslant\left|u+\alpha 2 a_{\beta} u+\alpha g_{\beta}(u)\right|_{2} \\
& \left(I+\alpha 2 a_{\beta} I+\alpha g_{\beta}\right)\left(a \tilde{K}_{2}^{r}\right) \supset a \tilde{K}_{2}^{r}
\end{aligned}
$$

(iv) we have $D_{g} \supset \widetilde{K}_{\mathrm{a}}$ and $\lim _{\beta \rightarrow 0^{+}}\left|a_{\beta} u+g_{\beta}(u)-a u-g(u)\right|_{1}=0$ uniformly on bounded subsets of $Y_{\mathrm{z}}$.

Proof. - It suffices to show (ii) and (iii). Let $u, v \in \widetilde{K}_{2}^{r}$; we have

$$
\begin{aligned}
& \left\langle 2 a_{\beta}(u-v)+g_{\beta}(u)-g_{\beta}(v), \operatorname{sgn}(u-v)\right\rangle_{1}= \\
& =2 \int a_{\beta}|u-v| d x+\frac{1}{2}\left[\int|u-v| d x \int(u+v) a_{\beta} d y+\int(u+v) \operatorname{sgn}(u-v) d x \int(u-v) a_{\beta} d y\right] \\
& -\frac{1}{2}\left[\int|u-v| a_{\beta} d x \int(u+v) d y+\int(u+v) \operatorname{sgn}(u-v) a_{\beta} d x \int(u-v) d y\right] \geqslant 0
\end{aligned}
$$

which proves (ii).

To prove (ii) take $v \in K_{2}^{r}$ and define

$$
\begin{aligned}
& u_{1}=v, \\
& \cdots \cdots \cdots \\
& u_{n}=\frac{v}{1+\alpha 2 a \beta+\alpha\left(u_{n-1}\right)^{-1}\left[g_{\beta}^{+}\left(u_{n-1}\right)-g_{\beta}^{-}\left(u_{n-1}\right)\right]} .
\end{aligned}
$$

We have $\left|u_{n}\right|_{1} \leqslant|v|_{1},\left|u_{n}\right|_{2} \leqslant|v|_{2}$ hence, in view of (i) there exsists $u=\lim u_{n} \in \tilde{K}_{2}^{r}$ and we have

$$
u+\alpha 2 a_{\beta} u+\alpha g_{\beta}(u)=v .
$$

Now let $u \in K_{2}$; we have

$$
\begin{aligned}
\left\langle 2 a_{\beta} u+g_{\beta}(u), \operatorname{sgn} u\right\rangle_{2}=\int|u|\left(1+|x|^{2}\right) 2 a_{\beta} d x+ & \\
& \quad-\int\left(1+|x|^{2}\right) a_{\beta}|u| d x \int u d y+\int\left(1+\left.|x|\right|^{2}\right)|u| d x \int a_{\beta} u d y \geqslant 0,
\end{aligned}
$$

hence combining these results the conclusion follows.

23 - Annali di Matematica 
As a consequence of Lemma 2.10.2 and Theorem 1.3.5 we have:

Theorem 2.10.2. - Assume that $u_{0} \geqslant 0$ a.e. $x \in R^{n},\left|u_{0}\right|_{1}<1$ and $u_{0} \in Y_{2}$. Then for every $T>0$ and $w \in O\left(0, T ; K_{2}^{r}\right)$ such that $\sup |w(t)|_{1} \leqslant 1$ the initial value problem (4) has a unique strong solution $u$. $\in \in[0, T]$

\subsection{1. - The Boltzmann equation.}

As an application of the results obtained in section 2.9 we shall study the Boltzmann equation in the space-independent case:

$$
\frac{\partial u(\xi, t)}{\partial t}+c \cdot \nabla_{\xi} u+g(u, u)=0, \quad \xi \in R^{3}, t>0,
$$

where $c=\left(e_{1}, c_{2}, c_{3}\right)$ is constant and different from zero $\left({ }^{4}\right)$ and the bilinear funetion $(u, v) \rightarrow g(u, v)$ is defined as follows $\left(^{5}\right)$

$$
g(u, v)=g^{+}(u, v)-g^{-}(u, v)
$$

where

$$
g^{+}(u, v)=\frac{1}{2} \int_{R^{3}} \int_{0}^{2 \pi} \int_{0}^{\pi / 2}\left[u(\xi) v\left(\xi_{1}\right)+u\left(\xi_{1}\right) v(\xi)\right] B\left(\theta, \xi, \xi_{1}\right) d \theta d \sigma d \xi_{1}
$$

and

$$
g^{-}(u, v)=\frac{1}{2} \int_{\mathbb{R}^{2}} \int_{0}^{2 \pi} \int_{0}^{\pi / 2}\left[u\left(\xi^{\prime}\right) v\left(\xi_{1}^{\prime}\right)+u\left(\xi_{1}^{\prime}\right) v\left(\xi^{\prime}\right)\right] B\left(\theta, \xi, \xi_{1}\right) d \theta d \sigma d \xi_{1}
$$

Here the variables $\xi^{\prime}$ and $\xi_{1}^{\prime}$ depend on $\xi, \xi_{1}$ through

$$
\begin{aligned}
& \xi^{\prime}=\xi+\alpha\left(\alpha \cdot\left(\xi-\xi_{1}\right)\right) \\
& \xi_{1}^{\prime}=\xi_{1}-\alpha\left(\alpha \cdot\left(\xi-\xi_{1}\right)\right)
\end{aligned}
$$

where $\alpha=(\cos \theta, \sin \theta \cos \sigma, \sin \theta \sin \sigma)$. These equations imply the following equalities

$$
\begin{aligned}
& \xi+\xi_{1}=\xi^{\prime}+\xi_{1}^{\prime}, \\
& \xi^{2}+\xi_{1}^{2}=\xi^{\prime 2}+\xi_{1}^{\prime 2} .
\end{aligned}
$$

(4) The case $c=(0,0,0)$ is treated in [10].

(5) By $u, v$ we denote measurable functions. 
$\left.B\left(0, \xi, \xi_{1}\right):\right] 0, \pi / 2\left[\times R^{6} \rightarrow R\right.$ is a given measurable function non negative a.e. such that

$$
\begin{aligned}
& B\left(\theta, \xi, \xi_{1}\right)=B\left(\theta, \xi_{1}, \xi\right) \\
& B\left(\theta, \xi, \xi_{1}\right)=B\left(\theta, \xi^{\prime}, \xi_{1}^{\prime}\right)
\end{aligned}
$$

Finally we recall that $g$ satisfies the following equalities (see [5] and [12]):

(5)

$$
\begin{aligned}
& \int g(u, v) d \xi=0, \\
& \int \xi^{2} g(u, v) d \xi=0 .
\end{aligned}
$$

We shall study the Boltzmann equation under the following assumption

$$
\sup _{\xi, \xi_{1} \in \mathbb{R}^{3}} \int_{0}^{\pi / 2} \frac{B\left(\theta, \xi, \xi_{1}\right)}{1+\sqrt{\xi^{2}+\xi_{1}^{2}}} d \theta=B<+\infty
$$

For each $\beta \in] 0,1]$ we shall consider the approximating function

$$
g_{\beta}(u, v)=g_{\beta}^{+}(u, v)-g_{\beta}^{-}(u, v)
$$

where

$$
\left.g_{\beta}^{+}(u, v)=\frac{1}{2} \iiint\left[u(\xi) v \xi_{1}\right)+u\left(\xi_{1}\right) v(\xi)\right] \frac{B\left(\theta, \xi, \xi_{1}\right)}{1+\beta \sqrt{\xi^{2}+\xi_{1}^{2}}} d \theta d \sigma d \xi_{1}
$$

and

$$
g_{\bar{\beta}}(u, v)=\frac{1}{2} \iiint\left[u\left(\xi^{\prime}\right) v\left(\xi_{1}^{\prime}\right)+u\left(\xi_{1}^{\prime}\right) v\left(\xi^{\prime}\right)\right] \frac{B\left(\theta, \xi, \xi_{1}\right)}{1+\beta \sqrt{\xi^{2}+\xi_{1}^{2}}} d \theta d \sigma d \xi_{1}
$$

We now quote a number of properties of the functions $g_{\beta}$ which are proved in [10]. By $g_{0}$ we denote the function $g$.

Lemma 2.11.1. - For each $\beta \in] 0,1]$ we have

$$
\int\left|g_{\beta}(u, v)\right|\left(1+\xi^{2}\right)^{i} d \xi \leqslant M_{\beta} \int|u|\left(1+\xi^{2}\right)^{i} d \xi \int|v|\left(1+\xi^{2}\right)^{i} d \xi, \quad i=0,1,2
$$

and

$$
\int|g(u, v)|\left(1+\xi^{2}\right)^{i} d \xi \leqslant M \int|u|\left(1+\xi^{2}\right)^{i+1} d \xi \int|v|\left(1+\xi^{2}\right)^{i+1} d \xi, \quad i=0,1
$$


where

$$
M_{\beta}=8 \pi \sup _{\xi, \xi_{i} \in \boldsymbol{R}^{s}} \int_{0}^{\pi / 2} \frac{B\left(\theta, \xi, \xi_{1}\right)}{1+\beta \sqrt{\xi^{2}+\xi_{1}^{2}}} d \theta
$$

and $M=8 \pi B$

LFMMA 2.11.2. - If $u$ is non negative a.e. we have

$$
\int\left(1+\xi^{2}\right)^{2} g_{\beta}(u, u) d \xi \geqslant-M \int\left(1+\xi^{2}\right) u d \xi \int\left(1+\xi^{2}\right)^{2} u d \xi
$$

for each $\beta \in[0,1]$.

LEMMA 2.11.3. - If $u$ and $v$ are any functions non negative a.e. then

$\int\left(1+\xi^{2}\right) \operatorname{sgn}(u-v)\left[g_{\beta}(u, u)-g_{\beta}(v, v)\right] d \xi \geqslant-2 M \int\left(1+\xi^{2}\right)^{2}(u+v) d \xi \int\left(1+\xi^{2}\right)|u-v| d \xi$ for each $\beta \in[0,1]$.

LEMma 2.11.4. - For each $\beta, \gamma \in[0,1]$ we have

$$
\int\left(1+\xi^{2}\right)^{i}\left|g_{\beta}(u, u)-g_{\gamma}(u, u)\right| d \xi \leqslant 8 M|\beta-\gamma|\left(\int\left(1+\xi^{2}\right)^{i+1}|u| d \xi\right)^{2}
$$

where $i=0,1$.

We will study the following Cauchy problem

$$
\left\{\begin{array}{l}
\frac{\partial u(\xi, t)}{\partial t}+c \cdot \nabla_{\xi} u+g(u, u)=0 \\
u(0, \xi)=u_{0}(\xi)
\end{array} \quad t \in[0, T]\right.
$$

and will give conditions on $u_{0}$ guaranteeing the existence of a unique strong solution of (7).

Let $\bar{Y}_{i}(i=0,1,2)$ be the Banach space of measurable functions $u: R^{3} \rightarrow R$ such that

$$
|u|_{i}=\int\left(1+\xi^{2}\right)^{i}|u| d \xi<+\infty
$$

equipped with the norm $\|_{i}$. By $K_{i} \subset Y_{i}$ we denote the closed convex set

$$
K_{i}=\left\{u \in Y_{i}, u \geqslant 0 \text { a.e. }|u|_{1} \leqslant N\right\}
$$


Let $A_{i}$ be the function defined as follows

$$
\left\{\begin{array}{l}
D_{A_{t}}=\left\{u \in Y_{i}, c \cdot \nabla_{\xi} u \in Y_{i}\right\}, \\
A_{i} u=e \cdot \nabla_{\xi} u .
\end{array}\right.
$$

It is well known that $-A_{1}$ is the infinitesimal generator of the linear semigroup $\exp \left[-t A_{1}\right]$ given by

$$
\left(\exp \left[-t A_{1}\right] u\right)(\xi)=u(\xi-c t)
$$

moreover it is easy to see that

$$
\begin{array}{ll}
\left|\exp \left[-t A_{1}\right] u\right|_{1} \leqslant \exp [2|c| t]|u|_{1}, & \text { for each } u \in Y_{1}, \\
\left|\exp \left[-t A_{1}\right] u\right|_{2} \leqslant \exp [4|c| t]|u|_{2}, & \text { for each } u \in Y_{2} .
\end{array}
$$

For each $\beta \in[0,1]$ let us denote by $g_{\beta}^{i}(i=0,1)$ the function defined as follows

$$
\begin{aligned}
& \left\{\begin{array}{l}
D_{g_{\theta}^{i}}=K_{i+1}, \\
\left(g_{0}^{i}(u)\right)(t)=g(u(t), u(t)),
\end{array}\right. \\
& \left\{\begin{array}{l}
D_{g_{\beta}^{i}}=K_{i}, \\
\left.\left.\left(g_{\beta}^{i}(u)\right)(t)=g_{\beta}(u(t), u(t)), \quad \beta \in\right] 0,1\right] .
\end{array}\right.
\end{aligned}
$$

By Lemmas 2.11.1, 2.11.2, 2.11.3, and 2.11.4 we get the following result:

Lemara 2.11.5. - The following statements hold:

(i) for each $\beta \in] 0,1] g_{\beta}^{i}$ is Lipschitz continuous on bounded subsets of $K_{i}$;

(ii) for each $u, v \in K_{2}^{r}$ we have

$$
|u-v|_{1} \leqslant\left|u-v+\alpha 8 M r(u-v)+\alpha g_{\beta}^{1}(u)-\alpha g_{\beta}^{1}(v)\right|_{1}
$$

(iii) for each $u \in K_{\mathbf{2}}$ we have

$$
|u|_{2} \leqslant \mid u+\alpha M N u+\alpha g_{\beta}^{1}(u)_{2}
$$

(iv) we have $\lim \left|g_{\beta}^{1}(u)-g^{1}(u)\right|_{1}=0$ uniformly on bounded subsets of $K_{\mathrm{a}}$ in $Y_{2}$;

(v) if $u_{n}, u \in K_{1}$ and $\lim \left|u_{n}-u\right|_{1}=0$ then $\lim \left|g^{1}\left(u_{n}\right)-g^{1}(u)\right|_{0}=0$.

The following lemma establish further properties of $g_{\beta}^{1}$.

Lemma 2.11.6. - For each $\beta \in] 0,1]$ there exists $\alpha(\beta)$ such that if $\alpha<\alpha(\beta)$ then

$$
\left(1-\alpha 8 M r I-\alpha g_{\beta}^{1}\right)\left(K_{2}^{r}\right) \subset K_{2}^{r} .
$$


Proof. - Let $u \in K_{2}^{q}, \alpha(\beta)=\left(8 M r+r M_{\beta}\right)^{-1}$ and $\alpha<\alpha(\beta)$; we have

$$
u-\alpha 8 M r u-\alpha g_{\beta}^{1}(u)=u\left(1-\alpha 8 M r-\frac{\alpha}{u} g^{+}(u, u)\right)+\alpha g^{-}(u, u) \geqslant 0 \text { a.e. . }
$$

Thus using (5) and Lemma 2.11.2 we obtain

$$
\begin{aligned}
& \left|u-\alpha 8 M r u-\alpha g_{\beta}^{1}(u)\right|_{1}=|u|_{1}-\alpha 8 M r|u|_{1} \leqslant N \\
& \left|u-\alpha 8 M r u-\alpha g_{\beta}^{1}(u)\right|_{2} \leqslant|u|_{2}-\alpha 8 M r|u|_{2}+\alpha N M|u|_{2} \leqslant r .
\end{aligned}
$$

By Lemmas 2.11.5 and 2.11.6 and Theorem 2.9.4 (see Remark 2, Part I) we obtain the following result:

Theorem 2.11.1. - Let $u_{0} \in K_{2}$ and let $N>\left|u_{0}\right|_{1}$. Then for each $T>0$ there is a unique $u \in O\left(0, T ; K_{2}\right)$ such that there exists $\left\{u_{\beta}\right\}$ verifying

(i) $u_{\beta} \in C^{1}\left(0, T ; Y_{0}\right), u_{\beta}(t) \in D_{A_{1}}$ for each $t \in[0, T], u_{\beta}(0)=u_{0}$,

(ii) we have $\lim _{\beta \rightarrow 0^{+}} u_{\beta}=u$ in $C\left(0, T ; Y_{1}\right)$ and $\lim _{\beta \rightarrow 0^{+}} \frac{d u_{\beta}}{d t}+A u_{\beta}=-g(u)$ in $C\left(0, T ; Y_{0}\right)$.

\section{REFERENCES}

[1] V. BARBU, Continuous perturbations of non-linear m-aceretive operators in Banach spaces, Boll. U.M.I., 6 (1972), pp. 270-278.

[2] R. BodmeR, Zur Boltzmanngleichung, Zürich, Seminar für Theor. Phys. E.T.H.

[3] H. BRÉzis, Opérateurs maximaux monotones et semi-groupes de contraction dans les espaces de Hilbert, Math. Studies 5, North-Holland (1973).

[4] F. E. BRowder, Nonlinear accretive operators in Banach spaces, Bull. Am. Soc., 73 (1967), pp. 470-476.

[5] C. Cercignani, Mathematical methods in kinetic theory, N. Y., Plenum Press (1969).

[6] G. DA PRATo, Somme d'applications non-linéaires, Symposia Mathematica, VII (1971), pp. 233-268.

[7] G. DA PRATo: Applications croissantes et équations d'évolutions dans les espaces de Banach, to appear.

[8] G. Da PRATo - P. GRISVARD, Somme d'opérateurs linéaires et équations differentielles opérationnelles, J. Math. pures et appl., to appear.

[9] G. Di BLAsio, Strong solutions for Boltzmann equation in the spatially homogeneous case, Boll. U.M.I., 8 (1973), pp. 127-136.

[10] G. Dx BLAsIo, Differentiability of spatially homogeneous solutions of the Boltzmann equation in the non Maxwellian case, Comm. Math. Phys., 38 (1974), pp. 331-340.

[11] G. Di Blasio, Somme d'opérateurs non linéaires et application à l'équation de Boltzmann, C. R. Acad. Sc., 280, série A (1975), pp. 1121-1123.

[12] H. GraD, Prineiples of kinetic theory of gases, Handbuch der Physik, 12, Berlin - Göt. tingen - Heidelberg, Springer (1958). 
[13] M. IANNELLI, A note on some non-linear non contraction semigroup, Boll. U.M.I., 6 (1970), pp. 1015-1025.

[14] T. KaTo, Nonlinear semigroups and evolution equations, J. Math. Soc. Japan, 19 (1967), pp. $508-520$.

[15] T. Kato, Accretive operators and nonlinear evolution equations in Banach spaces, Proo. Symp. Pure Math., 18, Part I, A.M.S. Providence, R. I. (1968), pp. 138-161.

[16] J. L. Lions, Quelques méthodes de résolution des problèmes aux limites non linéaires, DunodGauthier Villars (1969).

[17] R. H. MARTin Jr., Differential equations on closed subsets of a Banach space, Trans. Amer. Math. Soc., 179 (1973), pp. 399-414.

[18] R. H. MARTIN Jr., Invariant sets for perturbed semigroups of linear operators, to appear.

[19] G. WEBB, Nonlinear perturbations of linear accretive operators in Banach spaces, Journal of Funct. An., 10 (1972), pp. 191-203.

[20] A. JA. Povzner, The Boltzmann equation in the kinetic theory of gases, Mat. Sbornik, 58 (1962), pp. 65-86; translated in A.M.S. Translation, Series 2, 47 (1965), pp. 193-216. 